\title{
Synthetic Phase II Shewhart-type Attributes Control Charts When Process Parameters are Estimated
}

\author{
Philippe Castagliola, ${ }^{a}{ }^{\dagger}$ Shu Wu, a,b Michael B. C. Khoo ${ }^{c}$ \\ and S. Chakrabortid,e
}

\begin{abstract}
The performance of attributes control charts (such as $\mathrm{c}$ and $\mathrm{np}$ charts) is usually evaluated under the assumption of known process parameters (i.e., the nominal proportion of nonconforming units or the nominal number of nonconformities). However, in practice, these process parameters are rarely known and have to be estimated from an in-control phase I data set. In this paper, we derive the run length properties of the phase II synthetic $c$ and $n p$ charts with estimated parameters, and we investigate the number $\boldsymbol{m}$ of phase I samples that would be necessary for these charts in order to obtain similar in-control average run lengths as in the known parameters case. We also propose new specific chart parameters that allow these charts to have approximately the same in-control average run lengths as the ones obtained in the known parameter case. Copyright $\odot 2013$ John Wiley \& Sons, Ltd.
\end{abstract}

Keywords: phases I and II; attribute control chart; synthetic chart; binomial; Poisson; unknown parameter; average run length; Markov chain

\section{Introduction}

$\mathrm{B}$ ecause of the global market that considers customer satisfaction as a primary objective, quality has been established as a key competitive priority in the world of business. Statistical Process Control (SPC) is a collection of statistical techniques providing a rational management of a manufacturing process, which allows high-quality final products to be produced. Among the statistical process control tools, control charts are undeniably the most widely used for identifying changes in processes. When the considered quality characteristic consists of qualitative information, like the nominal proportion of nonconforming units or the nominal number of nonconformities, traditional variable control charts like the $\bar{X}, S$, and $R$ charts cannot be used and must be replaced by attributes control charts like the $n p$ or the $c$ charts (see, for instance, Montgomery ${ }^{1}$ ). Control charts have important applications, and they are particularly useful in service industries and in non-manufacturing quality-improvement efforts because many of the quality characteristics found in these environments are not easily measured on a numerical scale.

Shewhart-type control charts (for variables or attributes) are known to be rather inefficient in detecting small or moderate changes in a process compared with more advanced approaches such as the run rules charts, the adaptative charts (i.e., variable sampling interval and variable sampling size), and the exponentially weighted moving average (EWMA) and cumulative sum (CUSUM) charts. Among these more advanced approaches, it is worth to mention the synthetic chart, introduced by Wu and Spedding, ${ }^{2}$ that combines the Shewhart $\bar{X}$ chart and the conforming run length (CRL) chart. Under the normal distribution, the synthetic chart provides significantly better detection power than the basic $\bar{X}$ chart for all levels of mean shift and the EWMA and joint $\bar{X}$-EWMA charts for a mean shift $\delta$ larger than 0.8 . Wu and Spedding ${ }^{3}$ also provided a program in $C$ for an optimal design of a synthetic chart. Calzada and Scariano ${ }^{4}$ studied the robustness of the synthetic chart to non-normality. Davis and Woodall ${ }^{5}$ presented a Markov chain model of the synthetic chart and used it to evaluate the zero-state and steady-state average run length (ARL) performances. Scariano and Calzada ${ }^{6}$ worked on a synthetic control chart for exponential data. Sim $^{7}$ discussed combined $\bar{X}$ and CRL charts assuming that the quality characteristic follows a gamma distribution. Synthetic charts for process dispersion were proposed by Chen and Huang ${ }^{8}$ by merging the sample range, $R$ chart with the CRL chart, and Huang and Chen, ${ }^{9}$ by combining the sample standard deviation, $S$ with

\footnotetext{
${ }^{a}$ Université de Nantes, IRCCYN UMR CNRS 6597, LUNAM Université, Nantes, France

${ }^{b}$ School of Logistics Engineering, Wuhan University of Technology, Wuhan, China

'School of Mathematical Sciences, Universiti Sains Malaysia, Penang, Malaysia

${ }^{d}$ Department of Information Systems, Statistics, and Management Science, University of Alabama, Tuscaloosa, Alabama, USA

${ }^{e}$ Department of Statistics, University of Pretoria, Pretoria, South Africa

*Correspondence to: Philippe Castagliola, LUNAM Université, Université de Nantes, IRCCyN UMR CNRS 6597, Nantes, France.

'E-mail: Philippe.Castagliola@univ-nantes.fr
} 
the $C R L$ chart. Chen and Huang ${ }^{10}$ suggested a synthetic chart as well as its variable sampling interval schemes by integrating the Max and CRL charts. Costa and Rahim ${ }^{11}$ proposed a synthetic chart based on the non-central chi-square statistics, where the chart can detect a shift more effectively than the joint $\bar{X}$ and $R$ chart. Bourke ${ }^{12}$ re-evaluated the performance of a synthetic chart for detecting increases in fraction nonconforming by comparing it with the $n p$ chart, CRL-CUSUM chart and $R L_{2}$ chart, that is based on the moving sum of two successive CRLs. Khoo et $a l^{13}$ and Castagliola and Khoo ${ }^{14}$ proposed synthetic charts for skewed populations based on the weighted variance and scaled weighted variance methods, respectively. Aparisi and de Luna ${ }^{15}$ developed a synthetic chart, which does not detect shifts in a region of admissible shifts but detects shifts that are considered important. (Admissible shifts are shifts smaller than a given magnitude that are not desired to be detected or, at least, to delay as far as possible this detection. Contrarily, important shifts are shifts larger than a given magnitude that are desired to be detected.) Scariano and Calzada ${ }^{16}$ suggested generalized synthetic charts by considering the EWMA and CUSUM charts. Very recently, Calzada and Scariano ${ }^{17}$ suggested a synthetic control chart for monitoring the coefficient of variation, and Zhang et al ${ }^{18}$ investigated the synthetic $\bar{X}$ chart with estimated parameters. The growing interest in synthetic charts may be explained by the fact that many practitioners prefer waiting until the occurrence of a second point beyond the control limits before looking for an assignable cause.

An indispensable assumption for the development of control charts is that the process parameters are assumed known. In practice, the process parameters are rarely known. The process parameters are usually estimated from an in-control historical data set (phase I). As explained in Chakraborti et al. ${ }^{19}{ }^{19}$ (...) obtaining the reference sample is accomplished using an iterative procedure in Phase I in which trial limits are first constructed from the data (several subgroups or a number of observations), and then some charting statistics are placed on a control chart (...) subgroups corresponding to the charting statistics which are located outside the control limits are generally considered OOC (Out-of-Control) and removed from the analysis, and new limits are estimated from the remaining data. This step is repeated until no more charting statistics appear OOC so that the remaining data is taken to be IC (In-Control), at which point the final control limits may be constructed for Phase II monitoring. In our case, the control chart used during the phase I is either a Shewhart c or an $n p$ chart.

When the parameters are estimated, the performance of the control charts differs from the known parameters case because of the variability of the estimators used during the phase I. Recently, some authors have studied the impact of the estimation of the incontrol process parameters on the properties of the corresponding control charts. For a literature review on control charts with estimated parameters, see Jensen et al. ${ }^{20}$ Most of these authors have focused on location-type (i.e., $\bar{X}$ ) control charts: Quesenberry, ${ }^{21}$ Del Castillo, ${ }^{22}$ Chen, ${ }^{23}$ Ghosh et al., ${ }^{24}$ Jones et al., ${ }^{25}$ Nedumaran and Pignatiello, Jr., ${ }^{26}$ Jones, ${ }^{27}$ Yang et al., ${ }^{28}$ Champ and Jones, ${ }^{29}$ Chakraborti, ${ }^{30}$ Jensen et al., ${ }^{31}$ Zhang and Castagliola, ${ }^{32}$ and Zhang et al. ${ }^{18}$ Some other authors have focused on dispersion-type (i.e., $S, S^{2}$, and $R$ ) control charts: Hillier, ${ }^{33}$ Hawkins, $^{34}$ Quesenberry, ${ }^{35} \mathrm{Chen}^{36}$ Maravelakis et al., ${ }^{37}$ Maravelakis and Castagliola, $^{38}$ Castagliola et al., ${ }^{39}$ and Castagliola and Maravelakis. ${ }^{40}$

Very little work has been done concerning attributes control charts with estimated process parameters, as far as we know, and the only significant contributions were proposed by Braun, ${ }^{41}$ Chakraborti and Human ${ }^{42,43}$ and Castagliola and Wu. ${ }^{42}$ In these papers, the run length properties (i.e. $A R L$, standard deviation of the run length (SDRL), and cumulative distribution function (c.d.f.)) are derived for the $c$ and $p$ or $n p$ charts in the case where the parameters are estimated, and they are compared with that obtained in the case where these parameters are known. One of the main conclusions stated in Braun ${ }^{41}$ is '... as for variables charts, the effect of estimation on quantities, such as the average run length $(A R L)$ can be quite dramatic ...'

The goal of this paper is thus to extend the contributions of Braun ${ }^{41}$; Chakraborti and Human, ${ }^{43,44}$ and Castagliola and Wu ${ }^{42}$ to the case of the synthetic $c$ and $n p$ control charts as follows:

- By deriving the run length properties of the investigated synthetic $c$ and $n p$ charts,

- By providing an analysis concerning the required number $m$ of phase I samples in order to have similar in-control ARLs in both the estimated and known parameters cases, and

- By proposing new chart parameters especially dedicated to the number of phase I samples used in practice.

It is worth mentioning that in the case where there is no available phase I sample, an alternative approach is to use the socalled Q-charts proposed by Quesenberry, ${ }^{35}$ which can be used to monitor processes from start-up and to overcome the problem of having to wait to collect an in-control reference sample. Although this approach seems to be appealing, it nevertheless has some drawbacks: (i) as pointed by Del Castillo, ${ }^{45}$ the assumption of a stable process at start-up in the absence of prior process knowledge is unrealistic; (ii) sample points are no longer plotted in the original scale of measurement, and shop floor personnel may prefer to work with plots made in the original familiar scale of measurement; and (iii) by transforming the data into independent and identically distributed $N(0,1) Q$ statistics, the ability to detect process shifts in the transformed data decreases because the transformation hides the shifts. Moreover, the synthetic $c$ and $n p$ charts are not meant to be used in short runs (or low-volume manufacturing environment) but are rather suitable to be implemented in production runs where a reliable in-control phase I dataset is available.

The rest of the paper is structured as follows: the synthetic $c$ and $n p$ charts will be defined in Section 2 , and their corresponding run length properties will be presented for the known parameters case. In Section 3, the run length properties of the synthetic $c$ and $n p$ charts with estimated parameters will be derived. A subsection will be devoted to show how the run length properties in the estimated parameters case are computed in practice. In Section 4, a numerical comparison between the known and the estimated parameters case will be conducted for both the synthetic $c$ and $n p$ charts, providing an insight into the practical number $m$ of phase I samples to be used and also providing new chart parameters to be used in the estimated parameters case that give similar in-control ARLs to that of the known parameters case. Then the final conclusions will be summarized in Section 5. 


\section{The synthetic $c$ and $n p$ charts with known parameters}

2.1. The synthetic $c$ chart with known parameter $c_{0}$

Let $\left\{Y_{i, 1}, \ldots, Y_{i, n}\right\}, i=1,2, \ldots$, be a sample of $n \geq 1$ independent random variables $Y_{i, j}$ such that $Y_{i}=\sum_{j=1}^{n} Y_{i, j} \sim P\left(c_{1}\right)$, that is, a Poisson distribution with parameter $c_{1}$, where $c_{1}$ is an out-of-control average number of nonconformities. Similar to the synthetic $\bar{X}$ chart developed by Wu and Spedding, ${ }^{2}$ the synthetic $c$ chart combines a $c$ sub-chart designed with two $c o n t r o l$ limits, $L C L_{c}$ and $U C L_{c}$ and a CRL sub-chart having a single lower control limit, $H_{c} \in\{1,2, \ldots\}$. The control limits, $L C L_{c}$ and $U C L_{c}$, of the $c$ sub-chart are

$$
\begin{gathered}
L C L_{c}=\left\lceil\max \left(0, c_{0}-K_{c} \sqrt{c_{0}}\right)\right\rceil, \\
U C L_{c}=\left\lfloor c_{0}+K_{c} \sqrt{c_{0}}\right\rfloor,
\end{gathered}
$$

where $\lceil\ldots\rceil$ and $\lfloor\ldots\rfloor$ denote the rounded up and rounded down integers, respectively, $c_{0}$ is the known in-control average number of nonconformities, and $K_{c}>0$ is a constant. The central line of the $c$ sub-chart is $C L_{c}=c_{0}$. The CRL is defined as the number of inspected samples between two consecutive nonconforming samples, inclusive of the nonconforming sample at the end. The idea of this chart is that the distribution of $C R L$ will change when the fraction of nonconforming in the $c$ sub-chart, $\theta=P\left(Y_{i} \notin\left[L C L_{c}, U C L_{c}\right]\right)$, changes; that is, the $C R L$ decreases as $\theta$ increases and increases as $\theta$ decreases. An example with three $C R L$ samples is displayed in Figure 1 , which shows how the CRL value is determined, assuming that a process starts at $t=0$, and the white and black dots represent conforming and nonconforming samples, respectively. Here, $C R L_{1}=5, C R L_{2}=2$, and $C R L_{3}=4$. If we assume that we have a sequence $Y_{j}, Y_{j+1}, \ldots, Y_{i-1}, Y_{i}$ where $Y_{j} \notin\left[L C L_{c}, U C L_{c}\right]$ and $Y_{k} \in\left[L C L_{c}, U C L_{c}\right]$ for $k \in\{j+1, j+2, \ldots, i-1\}$ and $Y_{i} \notin\left[L C L_{c}, U C L_{c}\right]$, then the synthetic $c$ chart considers the process as out-of-control at time $i$ if $C R L=i-j \leq H_{c}$. This is to say, the synthetic $c$ chart signals if and only if both the plotting statistics plot outside the control limits and the $C R L$ is less than or equal to the lower control limit $H_{c}$.

Similarly, a synthetic version of the popular $u$ chart (for the average number of nonconformities per unit) can also be proposed by assuming $Y_{i, j} \sim P\left(u_{1}\right)$, where $u_{1}$ is an out-of-control average number of nonconformities per unit, by defining $\bar{Y}_{i}=\frac{1}{n} \sum_{j=1}^{n} Y_{i, j}$ and by using the following control limits, $L C L_{u}$ and $U C L_{u}$ for the $u$ sub-chart:

$$
\begin{gathered}
L C L_{u}=\max \left(0, u_{0}-K_{u} \sqrt{\frac{u_{0}}{n}}\right), \\
U C L_{u}=u_{0}+K_{u} \sqrt{\frac{u_{0}}{n}}
\end{gathered}
$$

where $u_{0}$ is the known in-control average number of nonconformities per unit and $K_{u}>0$ is a constant.

\subsection{The synthetic $n p$ chart with known parameter $p_{0}$}

Let $\left\{Y_{i, 1}, \ldots, Y_{i, n}\right\}, i=1,2, \ldots$, be a sample of $n \geq 1$ independent random variables $Y_{i, j} \sim B\left(p_{1}\right)$, that is, a Bernoulli random variable of parameter $p_{1}$, where $p_{1}$ is an out-of-control proportion of nonconforming units. Let $Y_{i}=\sum_{j=1}^{n} Y_{i, j}$ be the number of nonconforming units in the sample $\left\{Y_{i, 1}, \ldots, Y_{i, n}\right\}$. Similar to the synthetic $c$ chart, the synthetic $n p$ chart combines a $n p$ sub-chart designed with two control limits, $L C L_{n p}$ and $U C L_{n p}$ and a CRL sub-chart having a single lower control limit, $H_{n p} \in\{1,2, \ldots\}$. The control limits, $L C L_{n p}$ and $U C L_{n p}$ of the $n p$ sub-chart are

$$
\begin{gathered}
L C L_{n p}=\left\lceil\max \left(0, n p_{0}-K_{n p} \sqrt{n p_{0}\left(1-p_{0}\right)}\right)\right\rceil, \\
U C L_{n p}=\left\lfloor n p_{0}+K_{n p} \sqrt{n p_{0}\left(1-p_{0}\right)}\right\rfloor,
\end{gathered}
$$

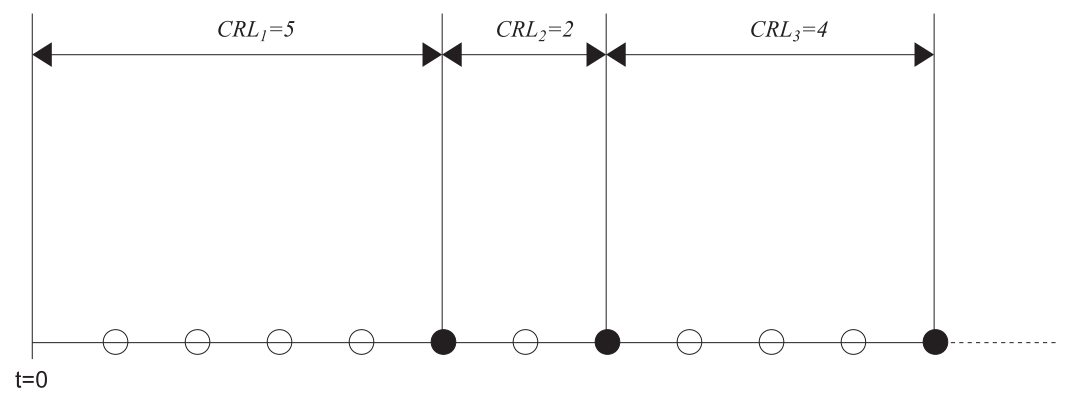

Conforming unit

- Nonconforming unit

Figure 1. Conforming run length 
where $p_{0}$ is the known in-control proportion of nonconforming units and $K_{n p}>0$ is a constant. The central line of the $n p$ sub-chart is $C L_{n p}=n p_{0}$. If we assume that we have a sequence $Y_{j}, Y_{j+1}, \ldots, Y_{i-1}, Y_{i}$ where $Y_{j} \notin\left[L C L_{n p}, U C L_{n p}\right]$ and $Y_{k} \in\left[L C L_{n p}, U C L_{n p}\right]$ for $k \in\{j+1, j+2$, $\ldots, i-1\}$ and $Y_{i} \notin\left[L C L_{n p}, U C L_{n p}\right]$, then the synthetic $n p$ chart considers the process as out-of-control at time $i$ if $C R L=i-j \leq H_{n p}$.

Similarly, a synthetic $p$ chart can also be proposed by defining $\bar{Y}_{i}=\frac{1}{n} \sum_{j=1}^{n} Y_{i, j}$ and by using the following control limits, $L C L_{p}$ and $U C L_{p}$ for the $p$ sub-chart:

$$
\begin{gathered}
L C L_{p}=\max \left(0, p_{0}-K_{p} \sqrt{\frac{p_{0}\left(1-p_{0}\right)}{n}}\right), \\
U C L_{p}=p_{0}+K_{p} \sqrt{\frac{p_{0}\left(1-p_{0}\right)}{n}},
\end{gathered}
$$

where $K_{p}>0$ is a constant.

\subsection{Choice of control chart parameters}

For an $\bar{X}$ chart with known process parameters $\left(\mu_{0}, \sigma_{0}\right)$, having $\mu_{0}-K \frac{\sigma_{0}}{\sqrt{n}}$ and $\mu_{0}+K \frac{\sigma_{0}}{\sqrt{n}}$ as control limits, it is common to choose $K=3$. This ensures an in-control $A R L_{0}=370.4$. As in Braun, ${ }^{41}$ the same choice, that is, $K=3$, is also commonly used for both the $c$ and $n p$ charts with known parameters $c_{0}$ and $p_{0}$ (but unfortunately, without ensuring a constant value for $A R L_{0}$, because of the discrete nature of the $c$ and $n p$ charts). This choice implicitly assumes that both the Binomial distribution and the Poisson distribution are well approximated by the normal distribution.

Our approach is based on an analog between the synthetic $\bar{X}$ chart and the synthetic $c$ and $n p$ charts. For the synthetic $\bar{X}$ chart with known process parameters $\left(\mu_{0}, \sigma_{0}\right)$, having $\mu_{0}-K \frac{\sigma_{0}}{\sqrt{n}}$ and $\mu_{0}+K \frac{\sigma_{0}}{\sqrt{n}}$ as control limits for the $\bar{X}$ sub-chart, there is an infinite number of couples $(H, K)$ that ensures a particular value for $A R L_{0}$. It is of course impossible to investigate all these couples. For this reason, we have decided to only select three specific couples, all of them corresponding to $n=5$ and $A R L_{0}=370.4$. These couples, dedicated to the synthetic $\bar{X}$ chart, have been chosen in order to optimally detect a specific shift $\delta$ in the mean:

- the couple $(H=47, K=2.639)$ is optimal for detecting a shift $\delta=0.25$, that is, a small shift;

- the couple $(H=7, K=2.322)$ is optimal for detecting a shift $\delta=0.75$, that is, a medium shift; and

- the couple $(H=2, K=2.085)$ is optimal for detecting a shift $\delta=1.5$, that is, a large shift.

Consequently, in the remainder of this paper, we also suggest to adopt these values as references for the chart parameters $\left(H_{c}, K_{c}\right)$ and $\left(H_{n p}, K_{n p}\right)$ for the synthetic $c$ and $n p$ charts, respectively (for the synthetic $u$ and $p$ charts, the same values can also be chosen for $\left(H_{u}, K_{u}\right)$ and $\left.\left(H_{p}, K_{p}\right)\right)$.

\subsection{Run length properties}

In this section, we assume that both the in-control average number of nonconformities $c_{0}$ and the in-control proportion of nonconforming $p_{0}$ are known. Let $L C L$ and $U C L$ be the control limits of either the synthetic $c$ sub-chart or the synthetic $n p$ sub-chart (i.e., $L C L$ stands for either $L C L_{c}$ or $L C L_{n p}$, and $U C L$ stands for either $U C L_{c}$ or $U C L_{n p}$ ). Let $H$ be the lower limit of the $C R L$ sub-chart (i.e., $H$ stands for either $H_{c}$ or $H_{n p}$ ). In order to obtain the run length properties of the synthetic $c$ and $n p$ charts, the general Markov chain approach proposed by Davis and Woodall ${ }^{5}$ for the synthetic $\bar{X}$ chart can be used. As for run rules type charts, this method consists of defining a transition probability matrix $\mathbf{P}_{(H+2, H+2)}$ with exactly $H+1$ transient states and one absorbing state. Each transient state corresponds to a sequence of $H$ samples (i) with no nonconforming sample, (ii) with a nonconforming sample at the first sample, and (iii) with a nonconforming sample at the second sample, $\ldots,(H+1)$ with a nonconforming sample at the $H$ th sample. The matrix $\mathbf{P}_{(H+2, H+2)}$ has the following structure:

$$
\mathbf{P}=\left(\begin{array}{cc}
\mathbf{Q} & \mathbf{r} \\
\mathbf{0}^{T} & 1
\end{array}\right)=\left(\begin{array}{cccccc|c}
1-\theta & \theta & 0 & \cdots & \cdots & 0 & 0 \\
0 & 0 & 1-\theta & \ddots & & 0 & \theta \\
\vdots & & \ddots & \ddots & \ddots & \vdots & \vdots \\
\vdots & & & \ddots & 1-\theta & 0 & \vdots \\
0 & \cdots & \cdots & \cdots & 0 & 1-\theta & \theta \\
1-\theta & 0 & \cdots & \cdots & \cdots & 0 & \theta \\
\hline 0 & \cdots & \cdots & \cdots & \cdots & 0 & 1
\end{array}\right)
$$

where $\mathbf{0}_{(H+1,1)}=(0,0, \ldots, 0)^{T}, \mathbf{Q}_{(H+1, H+1)}$ is the matrix of transient probabilities, vector $\mathbf{r}_{(H+1,1)}$ satisfies $\mathbf{r}=1-\mathbf{Q} 1$ (i.e., row probabilities of $\mathbf{P}$ must sum to 1$)$ with $1_{(H+1,1)}=(1,1, \ldots, 1)^{T}$ and $\theta=P\left(Y_{i} \notin[L C L, U C L]\right)$. More specifically, we have for the synthetic $c$ chart

$$
\theta=1-F_{P}\left(U C L_{c} \mid C_{1}\right)+F_{P}\left(L C L_{c}-1 \mid C_{1}\right)
$$


where $F_{P}(x \mid \lambda)$ is the c.d.f. of the Poisson distribution with parameter $\lambda$, that is,

$$
F_{P}(x \mid \lambda)=\sum_{z=0}^{x} \frac{e^{-\lambda} \lambda^{z}}{z !}
$$

and for the synthetic $n p$ chart,

$$
\theta=1-F_{B}\left(U C L_{n p} \mid n, p_{1}\right)+F_{B}\left(L C L_{n p}-1 \mid n, p_{1}\right),
$$

where $F_{B}(x \mid n, p)$ is the c.d.f. of the binomial distribution with parameters $(n, p)$, that is,

$$
F_{B}(x \mid n, p)=\sum_{z=0}^{x}\left(\begin{array}{l}
n \\
z
\end{array}\right) p^{z}(1-p)^{n-z}
$$

Using this Markov chain approach allows one to compute the run length distribution as well as the run length moments of the synthetic $c$ and $n p$ charts. The run length probability mass function (p.m.f) $f_{R L}(\ell)$ and c.d.f. $F_{R L}(\ell)$ of the synthetic $c$ and $n p$ charts with known parameters are defined for $\ell=\{1,2, \ldots\}$ and are equal to

$$
\begin{gathered}
f_{R L}(\ell)=\mathbf{q}^{T} \mathbf{Q}^{\ell-1} \mathbf{r}, \\
F_{R L}(\ell)=1-\mathbf{q}^{T} \mathbf{Q}^{\ell} 1,
\end{gathered}
$$

where the vector $\mathbf{q}_{(H+1,1)}$ of initial probabilities associated with the transient states is equal to $\mathbf{q}=(0,1,0, \ldots, 0)^{T} ;$ that is, the initial state is the second one. Concerning the run length moments, the ARL and the SDRL of the synthetic $c$ and $n p$ charts are given by

$$
\begin{gathered}
A R L=\mathbf{q}^{T}(\mathbf{I}-\mathbf{Q})^{-1} 1, \\
S D R L=\sqrt{2 \mathbf{q}^{T}(\mathbf{I}-\mathbf{Q})^{-2} \mathbf{Q} 1-A R L^{2}+A R L} .
\end{gathered}
$$

Using direct methods, it can be proven (Calzada and Scariano ${ }^{17}$ ) that

$$
\begin{gathered}
A R L=\frac{1}{\theta\left(1-(1-\theta)^{H}\right)}, \\
S D R L=\sqrt{\frac{2-\theta}{\left(1-(1-\theta)^{H}\right) \theta^{2}}+\frac{\frac{1}{\theta^{2}}-2 \sum_{k=1}^{H} k(1-\theta)^{k-1}}{\left(1-(1-\theta)^{H}\right)^{2}}},
\end{gathered}
$$

where $\theta$ is calculated either from (10) or from (12), for the synthetic $c$ and $n p$ charts, respectively. The run length properties of the synthetic $u$ chart can be obtained by replacing $L C L_{c}$ and $U C L_{c}$ by $L C L_{u}$ and $U C L_{u}$, respectively, and by replacing $c_{1}$ by $n u_{1}$ in (10). Concerning the synthetic $p$ chart, the run length properties can be obtained by replacing, in (12), $L C L_{n p}$ and $U C L_{n p}$ by $L C L_{p}$ and $U C L_{p}$, respectively.

\section{Synthetic $c$ and $n p$ charts with estimated parameters}

\subsection{Synthetic $c$ chart with estimated parameter $c_{0}$}

We assume that we have a phase I data set consisting of $i=1, \ldots, m$ samples $\left\{X_{i, 1}, \ldots, X_{i, n}\right\}$ of size $n$. Let us assume that there is independence within and between samples, and $X_{i}=\sum_{j=1}^{n} X_{i, j} \sim P\left(c_{0}\right)$, that is, a Poisson distribution with parameter $c_{0}$. An estimator $\hat{c}_{0}$ of $c_{0}$ is

$$
\hat{c}_{0}=\frac{1}{m} \sum_{i=1}^{m} X_{i}=\frac{X}{m}
$$

where $X \sim P\left(m c_{0}\right)$, that is, a Poisson distribution with parameter $m c_{0}$ defined for $x \in\{0,1, \ldots\}$. When $c_{0}$ is estimated by $\hat{c}_{0}$, the control limits of the synthetic $c$ chart with estimated parameter becomes

$$
\begin{gathered}
\widehat{L C L}=\left\lceil\max \left(0, \hat{c}_{0}-K_{c} \sqrt{\hat{c}_{0}}\right)\right\rceil, \\
\widehat{U C L}_{c}=\left\lfloor\hat{c}_{0}+K_{c} \sqrt{\hat{c}_{0}}\right\rfloor
\end{gathered}
$$


Let $\hat{\theta}=P\left(Y_{i} \notin\left[\widehat{L C L}_{c}, \widehat{U C L} c\right] \mid X=x\right)$ be the probability that the number $Y_{i}$ of nonconformities in sample $\left\{Y_{i, 1}, \ldots, Y_{i, n}\right\}$ is outside the $C$ sub-chart control limits $\widehat{L C L}_{c}$ and $\widehat{U C L}_{c}$ conditionally to $X=x$. If we replace $\hat{c}_{0}$ by $\frac{x}{m}$ in $\widehat{L C L}_{c}$ and $\widehat{U C L}_{c}$ and use the condition $X=x$, we have

$$
\begin{aligned}
\hat{\theta}=1 & -P\left(Y_{i} \leq\left\lfloor\frac{x}{m}+K_{c} \sqrt{\frac{x}{m}}\right\rfloor\right) \\
& +P\left(Y_{i} \leq \max \left(0,\left\lceil\frac{x}{m}-K_{c} \sqrt{\frac{x}{m}}\right\rceil\right)-1\right),
\end{aligned}
$$

and because $Y_{i} \sim P\left(c_{1}\right)$, we have

$$
\hat{\theta}=1-F_{P}\left(\left\lfloor\frac{x}{m}+K_{c} \sqrt{\frac{x}{m}}\right\rfloor \mid c_{1}\right)+F_{P}\left(\max \left(0,\left\lceil\frac{x}{m}-K_{c} \sqrt{\frac{x}{m}}\right\rceil\right)-1 \mid c_{1}\right) .
$$

As $X \sim P\left(m c_{0}\right)$ is defined for $x=\{0,1, \ldots\}$, the (unconditional) run length p.m.f. $f_{R L}(\ell)$ and c.d.f. $F_{R L}(\ell)$ of the synthetic $c$ chart with estimated parameter $c_{0}$ are equal to

$$
\begin{gathered}
f_{R L}(\ell)=\sum_{x=0}^{\infty}\left(\left(\mathbf{q}^{T} \hat{\mathbf{Q}}^{\ell-1} \hat{\mathbf{r}}\right) \times f_{P}\left(x \mid m c_{0}\right)\right), \\
F_{R L}(\ell)=1-\sum_{x=0}^{\infty}\left(\left(\mathbf{q}^{T} \hat{\mathbf{Q}}^{\ell} 1\right) \times f_{P}\left(x \mid m c_{0}\right)\right),
\end{gathered}
$$

where $f_{P}(x \mid \lambda)=\frac{e^{-\lambda} \lambda^{x}}{x !}$ is the p.m.f. of the Poisson distribution with parameter $\lambda$ and where $\hat{\mathbf{Q}}$ and $\hat{\mathbf{r}}$ are matrix $\mathbf{Q}$ and vector $\mathbf{r}$ in (9), respectively, in which $\theta$ is replaced by $\hat{\theta}$ in (24). The (unconditional) ARL of the synthetic chart with estimated parameter $c_{0}$ is equal to

$$
A R L=\sum_{x=0}^{\infty}\left(\widehat{A R L} \times f_{P}\left(x \mid m c_{0}\right)\right)
$$

where $\widehat{A R L}$ is defined as in (18), in which $\theta$ is replaced by $\hat{\theta}$ in (24). Finally, the (unconditional) SDRL of the synthetic $c$ chart with estimated parameter $c_{0}$ is equal to

$$
S D R L=\sqrt{\sum_{x=0}^{\infty}\left(\left(\widehat{S D R L}^{2}+\widehat{A R L}^{2}\right) \times f_{P}\left(x \mid m c_{0}\right)\right)-A R L^{2}}
$$

where $\widehat{S D R L}$ is defined as in (19), in which $\theta$ is replaced by $\hat{\theta}$ in (24). It can be easily shown that all the previous equations also hold for the synthetic $u$ chart (as defined in (3) and (4)) with estimated parameters with the following differences: the constant $c_{1}$ in (24) must be replaced by $n u_{1}$, and the term $m c_{0}$ in $f_{P}\left(x \mid m c_{0}\right)$ must be replaced by $m n u_{0}$.

\subsection{Synthetic $n p$ chart with estimated parameter $p_{0}$}

We assume that we have a phase I data set composed of $i=1, \ldots, m$ samples $\left\{X_{i, 1}, \ldots, X_{i, n}\right\}$ of size $n$. Let us assume that there is independence within and between samples, and $X_{i, j} \sim B\left(p_{0}\right)$, that is, a Bernoulli random variable of parameter $p_{0}$. An unbiased estimator $\hat{p}_{0}$ of $p_{0}$ is

$$
\hat{p}_{0}=\frac{1}{m n} \sum_{i=1}^{m} \sum_{j=1}^{n} X_{i, j}=\frac{X}{m n}
$$

where $X \sim B\left(m n, p_{0}\right)$, that is, a binomial random variable of parameters $\left(m n, p_{0}\right)$ defined for $x \in\{0,1, \ldots, m n-1, m n\}$. When $p_{0}$ is estimated by $\hat{p}_{0}$, the control limits of the synthetic $n p$ sub-chart becomes

$$
\begin{aligned}
\widehat{L C L}_{n p} & =\left\lceil\max \left(0, n \hat{p}_{0}-K_{n p} \sqrt{n \hat{p}_{0}\left(1-\hat{p}_{0}\right)}\right)\right\rceil \\
\widehat{U C L}_{n p} & =\left\lfloor n \hat{p}_{0}+K_{n p} \sqrt{n \hat{p}_{0}\left(1-\hat{p}_{0}\right)}\right\rfloor
\end{aligned}
$$

Similar to the synthetic $c$ chart, let $\hat{\theta}=P\left(Y_{i} \notin\left[\widehat{L C L}_{n p},, \widehat{U C L}_{n p}\right] \mid X=x\right)$ be the probability that the number $Y_{i}$ of nonconforming units in sample $\left\{Y_{i, 1}, \ldots, Y_{i, n}\right\}$ is outside the $n p$ sub-chart control limits $\widehat{L C L}_{n p}$ and $\widehat{U C L}_{n p}$, conditionally to $X=x$. If we replace $\hat{p}_{0}$ by $\frac{x}{m n}$ in $\widehat{L C L}_{n p}$ and $\widehat{U C L}_{n p}$ and use the condition $X=x$, we have 


$$
\begin{aligned}
\hat{\theta}=1 & -P\left(Y_{i} \leq\left\lfloor\frac{x}{m}+K_{n p} \sqrt{\frac{x}{m}\left(1-\frac{x}{m n}\right)}\right\rfloor\right) \\
& +P\left(Y_{i} \leq \max \left(0,\left\lceil\frac{x}{m}-K_{n p} \sqrt{\frac{x}{m}\left(1-\frac{x}{m n}\right)}\right\rceil\right)-1\right),
\end{aligned}
$$

and because $Y_{i} \sim B\left(n, p_{1}\right)$, we have

$$
\hat{\theta}=1-F_{B}\left(\left\lfloor\frac{x}{m}+K_{n p} \sqrt{\frac{x}{m}\left(1-\frac{x}{m n}\right)}\right\rfloor \mid n, p_{1}\right)+F_{B}\left(\max \left(0,\left\lceil\frac{x}{m}-K_{n p} \sqrt{\frac{x}{m}\left(1-\frac{x}{m n}\right)}\right\rceil\right)-1 \mid n, p_{1}\right) .
$$

As $X \sim B\left(m n, p_{0}\right)$ is defined for $x=\{0,1, \ldots, m n-1, m n\}$, the (unconditional) run length p.m.f. $f_{R L}(\ell)$ and c.d.f. $F_{R L}(\ell)$ of the synthetic $n p$ chart with estimated parameter $p_{0}$ are equal to

$$
\begin{gathered}
f_{R L}(\ell)=\sum_{x=0}^{m n}\left(\left(\mathbf{q}^{T} \hat{\mathbf{Q}}^{\ell-1} \hat{\mathbf{r}}\right) \times f_{B}\left(x \mid m n, p_{0}\right)\right), \\
F_{R L}(\ell)=1-\sum_{x=0}^{m n}\left(\left(\mathbf{q}^{T} \hat{\mathbf{Q}}^{\ell} \mathbf{1}\right) \times f_{B}\left(x \mid m n, p_{0}\right)\right),
\end{gathered}
$$

where $f_{B}(x \mid n, p)=\left(\begin{array}{l}n \\ x\end{array}\right) p^{x}(1-p)^{n-x}$ is the p.m.f. of the binomial distribution with parameters $(n, p)$. The (unconditional) ARL of the synthetic $n p$ chart with estimated parameter $p_{0}$ is equal to

$$
A R L=\sum_{x=0}^{m n}\left(\widehat{A R L} \times f_{B}\left(x \mid m n, p_{0}\right)\right)
$$

and the (unconditional) SDRL of the synthetic $n p$ chart with estimated parameter $p_{0}$ is equal to

$$
S D R L=\sqrt{\sum_{x=0}^{m n}\left(\left(\overline{S D R L}^{2}+\widehat{A R L}^{2}\right) \times f_{B}\left(x \mid m n, p_{0}\right)\right)-A R L^{2}}
$$

It can be easily shown that all the previous equations also hold for the synthetic $p$ chart (as defined in (7) and (8)) with estimated parameters.

\subsection{Computational note}

The computation of the p.m.f, c.d.f., ARL, and SDRL of both the synthetic $c$ and $n p$ charts with estimated parameters involves summations over domains that can be very large (depending on $m$ and $n$ for the synthetic $n p$ chart) and can be even infinite (for the synthetic $c$ chart), see (25)-(28) and (31)-(34), respectively. The time required for the computation of these summations can be greatly reduced (while keeping a high accuracy) by noticing that the terms $f_{P}\left(x \mid m c_{0}\right)$ (for the synthetic $c$ chart) and $f_{B}\left(x \mid m n, p_{0}\right)$ (for the synthetic $n p$ chart) reach a maximum close to $m c_{0}$ (for the synthetic $c$ chart) and $m n p_{0}$ (for the synthetic $n p$ chart) and both converge to zero when they diverge from $m c_{0}$ (for the synthetic $c$ chart) and from $m n p_{0}$ (for the synthetic $n p$ chart). Consequently, we suggest replacing these summations over $\{0,1, \ldots, \infty\}$ (for the synthetic $c$ chart) and over $\{0,1, \ldots, m n\}$ (for the synthetic $n p$ chart) by summations over $\left\{x_{\min }, x_{\max }\right\}$ where,

- for the synthetic $c$ chart: $\left\{\begin{array}{c}x_{\min }=\max \left(0,\left\lfloor m c_{0}-a \sqrt{m c_{0}}\right\rfloor\right) \\ x_{\max }=\left\lceil m c_{0}+a \sqrt{m c_{0}}\right\rceil\end{array} ;\right.$ and

- for the synthetic $n p$ chart: $\left\{\begin{array}{c}x_{\min }=\max \left(0,\left\lfloor m n p_{0}-a \sqrt{m n p_{0}\left(1-p_{0}\right)}\right\rfloor\right) \\ x_{\max }=\left\lceil m n p_{0}+a \sqrt{m n p_{0}\left(1-p_{0}\right)}\right\rceil\end{array}\right.$

Here, $a$ is a constant ensuring that the terms $f_{P}\left(x_{\min } \mid m c_{0}\right)$ and $f_{P}\left(x_{\max } \mid m c_{0}\right)$ (for the synthetic $c$ chart) and $f_{B}\left(x_{\min } \mid m n, p_{0}\right)$ and $f_{B}\left(x_{\max } \mid m n\right.$, $p_{0}$ ) (for the synthetic $n p$ chart) are close enough to zero (in practice, these values are less than $10^{-20}$ ). Simulations have shown that using $a=10$ yields excellent results. The value $a=10$ will be used in the rest of the paper.

\section{Performance analysis}

\subsection{Synthetic $\mathrm{c}$ chart}

Because the in-control case is always the worst case when evaluating control charts with estimated parameters (i.e., the larger the magnitude of the out-of-control condition, the more similar are the results between the known and the estimated parameters cases), 
we will only confine our paper to the in-control case. In Tables I-III, we present the in-control ARL and SDRL for the synthetic $c$ chart, for $c_{0}=\{5,10, \ldots, 100\}$ and $m=\{10,20,50,100,200, \infty\}$ (results for the $u$ chart can be deduced from those of the $c$ chart by assuming that $\left.c_{0}=n u_{0}\right)$. The value $m=\infty$ corresponds to the known parameter case, and the other $m$ values correspond to the estimated parameters case. The selected control chart parameters $\left(H_{c}, K_{c}\right)$ are defined as in Section 2.3, that is, $\left(H_{c}=2, K_{c}=2.085\right)$ in Table I, $\left(H_{c}=7, K_{c}=2.322\right)$ in Table II, and $\left(H_{c}=47, K_{c}=2.639\right)$ in Table III. Several interesting remarks can be drawn from the analysis of these tables:

\begin{tabular}{|lcccccc|}
\hline \multicolumn{6}{|l}{ Table I. In-control ARL and SDRL values for $\left(H_{c}=2, K_{c}=2.085\right), c_{0}=\{5,10, \ldots, 100\}$ and $m=\{10,20,50,100,200, \infty\}$} \\
$c_{0}$ & $m=10$ & $m=20$ & $m=50$ & $m=100$ & $m=200$ \\
\hline 5 & $(608.8,1180.0)$ & $(613.4,1084.9)$ & $(529.4,800.1)$ & $(464.6,663.7)$ & $(404.3,535.8)$ \\
10 & $(361.7,513.5)$ & $(404.1,550.9)$ & $(434.2,559.5)$ & $(425.8,527.1)$ & $(399.8,467.8)$ & $(342.8,365.9)$ \\
15 & $(332.7,438.3)$ & $(370.4,463.1)$ & $(387.4,460.6)$ & $(398.6,468.8)$ & $(417.8,493.6)$ & $(690.3,724.4)$ \\
20 & $(315.3,401.0)$ & $(350.6,418.2)$ & $(378.4,432.6)$ & $(396.6,446.6)$ & $(419.0,465.6)$ & $(477.4,505.2)$ \\
25 & $(302.3,381.4)$ & $(337.9,397.8)$ & $(366.9,415.7)$ & $(382.3,426.2)$ & $(395.5,435.4)$ & $(418.7,444.6)$ \\
30 & $(299.6,374.2)$ & $(334.0,390.9)$ & $(360.5,402.3)$ & $(375.1,413.3)$ & $(386.9,422.7)$ & $(414.5,440.2)$ \\
35 & $(295.5,363.7)$ & $(329.5,383.9)$ & $(360.7,403.9)$ & $(373.9,413.0)$ & $(385.8,423.7)$ & $(439.7,466.3)$ \\
40 & $(295.4,362.9)$ & $(329.2,382.7)$ & $(359.7,404.0)$ & $(373.0,414.3)$ & $(382.9,423.7)$ & $(487.0,515.1)$ \\
45 & $(287.4,350.0)$ & $(324.3,373.4)$ & $(354.5,392.7)$ & $(365.7,398.3)$ & $(370.1,398.5)$ & $(269.1,289.3)$ \\
50 & $(287.0,348.6)$ & $(321.9,370.5)$ & $(354.2,394.1)$ & $(368.2,405.3)$ & $(375.1,410.8)$ & $(321.8,344.1)$ \\
55 & $(285.4,345.7)$ & $(321.0,367.0)$ & $(351.4,387.4)$ & $(364.1,396.2)$ & $(372.1,402.6)$ & $(388.3,413.2)$ \\
60 & $(284.8,345.3)$ & $(320.8,367.8)$ & $(351.3,389.1)$ & $(365.8,401.0)$ & $(373.4,407.6)$ & $(471.7,499.4)$ \\
65 & $(284.4,343.0)$ & $(319.2,364.4)$ & $(350.9,386.8)$ & $(364.7,397.8)$ & $(372.1,404.1)$ & $(313.9,335.9)$ \\
70 & $(282.9,341.8)$ & $(318.0,362.2)$ & $(349.2,382.9)$ & $(361.6,391.5)$ & $(369.5,397.7)$ & $(389.6,414.4)$ \\
75 & $(282.9,340.1)$ & $(318.9,364.0)$ & $(349.3,384.1)$ & $(362.5,394.1)$ & $(369.4,399.3)$ & $(484.1,512.2)$ \\
80 & $(279.2,335.8)$ & $(316.7,360.4)$ & $(349.3,384.5)$ & $(362.7,395.2)$ & $(370.4,401.8)$ & $(347.3,370.6)$ \\
85 & $(281.4,339.0)$ & $(317.5,361.5)$ & $(348.7,383.4)$ & $(362.8,395.0)$ & $(370.6,401.9)$ & $(435.4,461.9)$ \\
90 & $(282.0,337.6)$ & $(317.3,360.0)$ & $(348.1,381.8)$ & $(362.0,393.2)$ & $(369.7,400.1)$ & $(325.9,348.4)$ \\
95 & $(276.6,330.5)$ & $(313.9,355.8)$ & $(347.1,380.1)$ & $(361.2,391.6)$ & $(369.1,398.6)$ & $(410.4,436.0)$ \\
100 & $(277.7,332.5)$ & $(315.0,356.8)$ & $(346.5,379.1)$ & $(360.3,390.0)$ & $(368.0,396.8)$ & $(317.0,339.1)$ \\
\hline
\end{tabular}

\begin{tabular}{|lcccccc|}
\hline \multicolumn{6}{|l}{ Table II. In-control ARL and SDRL values for $\left(H_{c}=7, K_{c}=2.322\right), c_{0}=\{5,10, \ldots, 100\}$ and $m=\{10,20,50,100,200, \infty\}$} \\
$c_{0}$ & $m=10$ & $m=20$ & $m=50$ & $m=100$ & $m=200$ \\
\hline 5 & $(549.3,906.0)$ & $(531.5,793.3)$ & $(557.1,761.3)$ & $(597.1,778.2)$ & $(656.3,809.0)$ & $(793.5,859.4)$ \\
10 & $(401.8,627.9)$ & $(414.0,583.0)$ & $(432.3,569.3)$ & $(460.7,580.9)$ & $(480.2,569.1)$ & $(517.3,569.0)$ \\
15 & $(342.3,498.6)$ & $(368.4,498.6)$ & $(376.6,468.6)$ & $(370.0,434.7)$ & $(364.8,418.6)$ & $(211.0,241.3)$ \\
20 & $(327.8,459.4)$ & $(355.1,460.1)$ & $(373.4,452.3)$ & $(390.7,464.3)$ & $(407.8,474.1)$ & $(442.5,489.7)$ \\
25 & $(299.3,406.7)$ & $(339.2,436.7)$ & $(370.5,457.1)$ & $(381.4,461.4)$ & $(380.4,451.9)$ & $(344.3,385.0)$ \\
30 & $(299.0,400.4)$ & $(334.2,422.1)$ & $(365.1,440.8)$ & $(376.1,446.3)$ & $(376.1,442.0)$ & $(312.7,351.2)$ \\
35 & $(294.2,390.5)$ & $(329.6,412.3)$ & $(360.1,430.6)$ & $(373.3,439.0)$ & $(375.8,438.0)$ & $(310.8,349.2)$ \\
40 & $(288.7,381.4)$ & $(324.5,403.2)$ & $(355.5,423.7)$ & $(369.9,433.9)$ & $(374.7,435.6)$ & $(326.9,366.5)$ \\
45 & $(283.5,371.6)$ & $(319.3,394.4)$ & $(352.4,416.7)$ & $(365.9,425.8)$ & $(373.2,430.4)$ & $(357.1,398.8)$ \\
50 & $(284.6,372.2)$ & $(320.9,393.1)$ & $(350.6,410.2)$ & $(363.1,416.5)$ & $(371.2,421.5)$ & $(400.6,445.1)$ \\
55 & $(280.1,363.5)$ & $(317.8,388.7)$ & $(350.9,412.3)$ & $(365.6,423.3)$ & $(374.4,430.6)$ & $(458.0,506.2)$ \\
60 & $(278.9,361.3)$ & $(316.1,385.2)$ & $(347.3,404.5)$ & $(360.4,412.6)$ & $(366.9,414.9)$ & $(274.1,309.6)$ \\
65 & $(279.4,361.5)$ & $(315.7,384.3)$ & $(348.4,407.0)$ & $(362.8,418.2)$ & $(370.9,424.7)$ & $(327.1,366.7)$ \\
70 & $(275.5,354.3)$ & $(313.2,379.1)$ & $(344.7,399.5)$ & $(359.4,409.6)$ & $(367.5,415.2)$ & $(392.9,436.9)$ \\
75 & $(274.5,353.8)$ & $(312.0,378.3)$ & $(345.2,402.0)$ & $(360.3,413.5)$ & $(368.8,420.3)$ & $(474.2,523.4)$ \\
80 & $(273.5,351.4)$ & $(310.9,376.0)$ & $(346.0,401.6)$ & $(360.7,413.2)$ & $(369.1,420.1)$ & $(321.8,360.9)$ \\
85 & $(273.3,350.1)$ & $(311.0,375.2)$ & $(344.5,398.2)$ & $(358.5,408.2)$ & $(366.6,414.2)$ & $(394.6,438.8)$ \\
90 & $(274.2,352.0)$ & $(310.2,373.9)$ & $(343.6,397.4)$ & $(358.3,408.3)$ & $(366.0,413.7)$ & $(484.8,534.6)$ \\
95 & $(271.3,347.1)$ & $(310.6,374.6)$ & $(343.7,398.1)$ & $(358.8,410.1)$ & $(367.1,416.8)$ & $(349.3,390.4)$ \\
100 & $(271.5,348.0)$ & $(308.8,371.9)$ & $(343.2,397.0)$ & $(358.4,409.3)$ & $(367.4,417.1)$ & $(432.5,479.1)$ \\
\hline
\end{tabular}




\begin{tabular}{|llccccc|}
\hline \multicolumn{6}{|l}{ Table III. In-control ARL and SDRL values for $\left(H_{c}=47, K_{c}=2.639\right), c_{0}=\{5,10, \ldots, 100\}$ and $m=\{10,20,50,100,200, \infty\}$} \\
$c_{0}$ & $m=10$ & $m=20$ & $m=50$ & $m=100$ & $m=200$ \\
\hline 5 & $(2307.7,10789.4)$ & $(1044.7,5088.3)$ & $(492.5,1235.4)$ & $(409.2,738.0)$ & $(379.2,661.9)$ & $(153.1,197.6)$ \\
10 & $(463.3,867.7)$ & $(426.8,749.8)$ & $(419.3,684.2)$ & $(410.8,628.7)$ & $(404.2,557.6)$ & $(427.8,524.0)$ \\
15 & $(382.7,651.1)$ & $(391.3,622.3)$ & $(380.7,550.2)$ & $(384.4,530.3)$ & $(401.8,538.2)$ & $(502.5,609.9)$ \\
20 & $(340.7,551.7)$ & $(361.4,558.5)$ & $(373.9,544.7)$ & $(367.5,512.2)$ & $(353.7,479.3)$ & $(257.3,324.3)$ \\
25 & $(318.7,500.4)$ & $(351.0,520.1)$ & $(364.7,508.3)$ & $(369.8,498.1)$ & $(379.4,501.2)$ & $(493.3,599.4)$ \\
30 & $(301.7,461.3)$ & $(337.4,487.9)$ & $(358.1,492.2)$ & $(366.1,490.3)$ & $(373.1,489.1)$ & $(378.7,467.1)$ \\
35 & $(295.6,443.6)$ & $(329.7,469.4)$ & $(355.4,483.5)$ & $(363.1,483.3)$ & $(365.4,478.9)$ & $(330.3,410.6)$ \\
40 & $(289.8,433.7)$ & $(323.4,455.9)$ & $(351.1,471.9)$ & $(361.6,475.8)$ & $(364.4,473.6)$ & $(312.7,389.8)$ \\
45 & $(285.6,423.8)$ & $(321.5,449.7)$ & $(348.3,464.3)$ & $(360.2,470.9)$ & $(364.6,471.0)$ & $(312.8,390.0)$ \\
50 & $(282.7,418.7)$ & $(318.0,442.3)$ & $(345.9,459.7)$ & $(358.8,467.6)$ & $(364.2,469.0)$ & $(325.5,404.9)$ \\
55 & $(277.9,407.7)$ & $(312.8,432.8)$ & $(344.0,454.7)$ & $(356.3,461.9)$ & $(362.9,464.9)$ & $(348.6,432.1)$ \\
60 & $(277.8,406.5)$ & $(311.5,428.4)$ & $(342.5,449.2)$ & $(354.8,455.4)$ & $(361.5,458.0)$ & $(381.7,470.6)$ \\
65 & $(275.8,402.1)$ & $(310.1,424.8)$ & $(341.3,446.8)$ & $(355.6,456.9)$ & $(363.8,462.7)$ & $(425.2,521.0)$ \\
70 & $(271.9,394.7)$ & $(309.3,423.5)$ & $(341.8,447.5)$ & $(354.8,455.8)$ & $(361.4,458.8)$ & $(480.2,584.4)$ \\
75 & $(272.8,395.4)$ & $(308.2,419.9)$ & $(340.4,442.2)$ & $(353.5,450.8)$ & $(361.2,456.2)$ & $(297.1,371.5)$ \\
80 & $(270.2,391.1)$ & $(307.2,418.6)$ & $(339.8,442.6)$ & $(353.7,452.7)$ & $(361.5,458.1)$ & $(346.1,429.0)$ \\
85 & $(268.9,387.6)$ & $(306.3,415.8)$ & $(339.0,439.6)$ & $(353.3,450.3)$ & $(361.6,456.4)$ & $(405.7,498.5)$ \\
90 & $(269.0,388.1)$ & $(306.0,415.1)$ & $(338.9,439.5)$ & $(353.0,449.8)$ & $(360.2,453.9)$ & $(478.4,582.2)$ \\
95 & $(266.3,382.8)$ & $(303.9,411.2)$ & $(337.6,437.3)$ & $(353.3,450.2)$ & $(361.8,457.2)$ & $(326.8,406.5)$ \\
100 & $(266.3,382.5)$ & $(303.5,410.2)$ & $(338.0,436.3)$ & $(352.2,446.8)$ & $(360.3,452.5)$ & $(390.6,480.9)$ \\
\hline
\end{tabular}



- Because of the discrete nature of the synthetic c chart, it is impossible to have a common in-control $A R L_{0}$ value in the case where $m=\infty$ (i.e., known parameter case) for all the values of $c_{0}$ considered. The in-control $A R L$ values are sometimes smaller and sometimes larger than 370.4 (see the rightmost column of Tables I-III). This is a well known drawback of attributes charts in general.

- For a particular value of $c_{0}$, the in-control ARL values can be very different in the known and in the estimated parameter case. For instance, in Table I, if $c_{0}=5$, the in-control ARL is $A R L_{0}=342.8\left(S D R L_{0}=365.9\right)$ when $m=\infty$, whereas when $m=10$, we have $A R L_{0}=608.8$ 
$\left(S D R L_{0}=1180.0\right)$. When $m$ increases, the difference in terms of in-control ARLs, between the known and the estimated parameter case, tends to decrease but not always in a monotonic way (unlike $\bar{X}$-type charts where this difference always reduces monotonically with $m$ ).

- For $m<\infty$, depending on the value of $c_{0}$, the in-control ARL values are either smaller or larger than the in-control ARL values corresponding to $m=\infty$. In the previous example with $c_{0}=5$, the in-control ARL values corresponding to $m<\infty$ are all larger than the in-control ARL value $\left(A R L_{0}=342.8\right)$ corresponding to $m=\infty$. But, for instance, in Table I, case $c_{0}=20$, the corresponding incontrol $A R L$ values $\left(315.3,350.6,378.4,396.6\right.$, and 419.0) are smaller than the in-control $A R L$ value $\left(A R L_{0}=477.4\right)$ corresponding to $m=\infty$. This result (already emphasized in Braun ${ }^{41}$ ) is quite different from what can be observed in the case of $\bar{X}$-type charts where the in-control $A R L$ values corresponding to $m<\infty$ are always larger than the in-control ARL value corresponding to $m=\infty$.

Because the in-control ARL values are different in the known and in the estimated parameter case, an interesting question is how large the number $m$ of phase I samples should be in order to have approximatively the same in-control ARL values in both the known and estimated parameter cases if we keep, as in Tables I-III, the same control chart parameters $\left(H_{c}=2, K_{c}=2.085\right),\left(H_{c}=7, K_{c}=2.322\right)$ and $\left(H_{c}=47, K_{c}=2.639\right)$ ? In Table IV, we have computed, for $c_{0}=\{5,10, \ldots, 100\}$, the minimum values $m^{*}$ of $m$ satisfying $\Delta=\frac{\left|A R L_{0, m}+A R L_{0, \infty}\right|}{A R L_{0 . \infty}}<0.05$, that is, such that the relative difference between the in-control $A R L_{0, m^{*}}$ (estimated parameter case) and the in-control $A R L_{0, \infty}$ (known parameter case) is not larger than $5 \%$. As it can be noticed in Table IV:

- Depending on the value of $c_{0}$, the value of $m^{*}$ satisfying $\Delta<0.05$ can be very large and, in some cases, larger than 10,000 .

- When $c_{0}$ increases, there is no particular trend for $m^{*}$. For example, in the case $\left(H_{c}=2, K_{c}=2.085\right)$, if $c_{0}=5$, then we need at least $m^{*}=410$ samples in order to have $\Delta<0.05$. This value decreases down to $m^{*}=10$ if $c_{0}=10$ and then increases to $m^{*}=8110$ if $c_{0}=15$. This phenomenon has already been emphasized in the case of the $c$ chart in Castagliola and $\mathrm{Wu}^{42}$ and is due to the discrete nature of the control limits.

- For a particular value $c_{0}$, the selected chart parameters $\left(H_{c} K_{c}\right)$ have no influence on $m^{*}$. For example, if $c_{0}=5$, the value $m^{*}=410$ for $\left(H_{c}=2, K_{c}=2.085\right)$ is smaller than the value $m^{*}=740$ for $\left(H_{c}=7, K_{c}=2.322\right)$, but if $c_{0}=20$, this is the opposite with $m^{*}=510$ for $\left(H_{c}=2, K_{c}=2.085\right)$, which is larger than $m^{*}=340$ for $\left(H_{c}=7, K_{c}=2.322\right)$.

As presented in Castagliola and $\mathrm{Wu}^{42}$ for the same values of $c_{0}=\{5,10, \ldots, 100\}$, the rightmost column of Table IV lists the minimum values of $m^{*}$ for the Shewhart $c$ chart. As it can be noticed, there is no particular trend concerning which control chart requires a smaller number of samples. For instance, if $c_{0}=25$, then depending on the choice of $\left(H_{c}, K_{c}\right)$, the values of $m^{*}$ for the synthetic $c$ chart are 230, 20, and 1890, whereas for the Shewhart $c$ chart, this value is larger than 10,000. On the other hand, if $c_{0}=85$, then depending on the choice of $\left(H_{c} K_{c}\right)$, the values of $m^{*}$ for the synthetic $c$ chart are 2750,550 , and 1310, whereas for the Shewhart $c$ chart, this value reduces to 30 .

In conclusion, if we want to use the control chart parameters $\left(H_{c}=2, K_{c}=2.085\right),\left(H_{c}=7, K_{c}=2.322\right)$, and $\left(H_{c}=47, K_{c}=2.639\right)$ for the synthetic $c$ chart with estimated parameter $c_{0}$, having run length performances close to that of the synthetic $c$ chart with known parameter, then we must pay the price to obtain a large number $m$ of phase I samples, sometimes larger than 10,000 . But from a practical point of view, obtaining so many samples could be hard to handle in practice (i.e., completing the phase I chart implementation can take too long a time) and can be very costly. In order to relax this constraint on the number of phase I samples, we suggest to compute alternative chart parameters $\left(H_{c}^{\prime}{ }_{c} K_{c}^{\prime}\right)$, which take the value of $m$ into account and allow the in-control ARL value corresponding to the estimated parameter case to be as close as possible to the in-control ARL value corresponding to the known parameter case.

These values are in Tables V-VII for $c_{0}=\{5,10, \ldots, 100\}$ and for $m=\{10,20,50,100,200\}$. For example, in Table V, if $c_{0}=5$ and $m=10$, the chart parameters are $\left(H_{c}^{\prime}=84, K_{c}^{\prime}=2.49\right)$, and in this case, the in-control ARL is $A R L_{0}=342.8\left(S D R L_{0}=1102.2\right)$. Referring to Table I, the incontrol ARL corresponding to $m=\infty$ (i.e., the known parameter case) is also $A R L_{0}=342.8$. Consequently, the use of the new chart parameters $\left(H_{c}^{\prime}=84, K_{c}^{\prime}=2.49\right)$ instead of $\left(H_{c}=2, K_{c}=2.085\right)$ allows to obtain the same in-control ARL as for the known parameter case.

\subsection{Synthetic $n p$ chart}

Tables VIII-X are the counterparts of Tables I-III but for the synthetic $n p$ charts. In Table VIII, we present the in-control ARL and SDRL for the synthetic $n p$ chart for $n=\{25,50,75,100\}, p_{0}=\{0.01,0.02,0.05,0.1,0.15,0.2\}, m=\{10,20,50,100,200, \infty\}$, and chart parameters $\left(H_{n p}=\right.$ $\left.2, K_{n p}=2.085\right)$, whereas in Tables IX and X, the chart parameters are $\left(H_{n p}=7, K_{n p}=2.322\right)$ and $\left(H_{n p}=47, K_{n p}=2.639\right)$, respectively. Conclusions for Tables VIII-X are similar to those for Tables I-III; that is,

- There is no common in-control ARL value in the known parameter case for all the values of $n$ and $p_{0}$ considered;

- The in-control ARL values can be very different in the known versus estimated parameter case; and

- Depending on the values of $n$ and $p_{0}$, the in-control ARL values for $m<\infty$ are either smaller or larger than the in-control ARL values corresponding to $m=\infty$.

A simple example illustrating these remarks can be given, for instance, for the synthetic $n p$ chart with $\left(H_{n p}=2, K_{n p}=2.085\right)$, see Table VIII, when $n=75$ and $p_{0}=0.05$. For these values, the in-control $A R L$ is $A R L_{0}=449.7\left(S D R L_{0}=476.6\right)$ when $m=\infty$ and $A R L_{0}=714.5\left(S D R L_{0}=1521.3\right)$ when $m=10$. In this example, the in-control ARL corresponding to the estimated parameter case is larger than the one corresponding to the known parameter case. But if we choose a second example with $n=75, p_{0}=0.15$, the in-control $A R L$ is $A R L_{0}=411.5\left(S D R L_{0}=437.2\right)$ when $m=\infty$ and $A R L_{0}=345.8\left(S D R L_{0}=470.2\right)$ when $m=10$. In this second example, the in-control ARL corresponding to the estimated parameter case is smaller than the one corresponding to the known parameter case. 


\begin{tabular}{|c|c|c|c|c|c|}
\hline \multirow[b]{2}{*}{$c_{0}$} & \multicolumn{5}{|c|}{$\left(H^{\prime}{ }^{\prime}, K_{c}^{\prime}{ }^{\prime} A R L_{0}, S D R L_{0}\right)$} \\
\hline & $m=10$ & $m=20$ & $m=50$ & $m=100$ & $m=200$ \\
\hline 5 & $(84,2.49,342.8,1102.2)$ & $(69,2.51,342.8,1053.8)$ & $(87,2.67,342.9,833.9)$ & $(4,2.18,342.4,477.4)$ & $(95,2.74,342.9,514.3)$ \\
\hline 10 & $(39,2.57,364.7,696.7)$ & $(49,2.62,364.6,600.5)$ & $(63,2.65,364.7,624.0)$ & $(92,2.73,364.7,607.6)$ & $(45,2.60,364.6,492.4)$ \\
\hline 15 & $(24,2.64,690.5,1140.2)$ & $(25,2.65,690.4,1059.1)$ & $(82,2.84,690.2,1136.7)$ & $(95,2.87,690.3,1025.4)$ & $(70,2.84,690.7,940.0)$ \\
\hline 20 & $(36,2.66,477.2,770.9)$ & $(70,2.75,477.4,749.3)$ & $(44,2.68,477.6,682.9)$ & $(66,2.74,477.2,655.5)$ & $(60,2.72,477.4,644.2)$ \\
\hline 25 & $(55,2.71,418.7,666.5)$ & $(68,2.73,418.8,631.4)$ & $(55,2.69,418.7,593.7)$ & $(22,2.54,418.7,540.0)$ & $(75,2.74,418.5,575.2)$ \\
\hline 30 & $(40,2.67,414.2,624.9)$ & $(30,2.61,414.3,580.7)$ & $(15,2.48,414.6,528.6)$ & $(67,2.72,414.4,564.4)$ & $(62,2.71,414.5,548.7)$ \\
\hline 35 & $(94,2.81,439.6,691.7)$ & $(97,2.80,439.8,655.7)$ & $(92,2.78,439.7,620.9)$ & $(54,2.70,439.7,579.5)$ & $(17,2.51,439.7,530.2)$ \\
\hline 40 & $(98,2.84,487.0,768.8)$ & $(60,2.75,486.9,695.7)$ & $(32,2.64,486.9,632.9)$ & $(94,2.80,486.9,662.3)$ & $(64,2.74,486.9,627.8)$ \\
\hline 45 & $(62,2.67,269.2,405.8)$ & $(84,2.69,269.3,390.4)$ & $(6,2.24,269.1,316.9)$ & $(2,2.02,269.0,297.2)$ & $(3,2.04,249.1,276.6)$ \\
\hline 50 & $(43,2.65,321.8,470.5)$ & $(86,2.73,321.5,463.1)$ & $(9,2.35,321.9,385.8)$ & $(14,2.42,321.8,390.2)$ & $(89,2.71,321.8,430.3)$ \\
\hline 55 & $(44,2.69,388.2,566.6)$ & $(22,2.56,388.3,508.6)$ & $(91,2.76,388.2,531.6)$ & $(18,2.50,388.2,471.8)$ & $(28,2.57,388.4,478.4)$ \\
\hline 60 & $(51,2.75,471.7,687.2)$ & $(7,2.40,471.7,572.2)$ & $(27,2.61,471.7,592.3)$ & $(41,2.67,471.9,598.3)$ & $(20,2.55,471.3,565.2)$ \\
\hline 65 & $(19,2.52,313.9,430.2)$ & $(93,2.74,313.9,448.0)$ & $(74,2.69,314.0,423.9)$ & $(17,2.45,313.8,381.2)$ & $(28,2.53,313.9,386.7)$ \\
\hline 70 & $(20,2.57,389.6,532.5)$ & $(74,2.75,389.9,545.4)$ & $(9,2.39,389.5,457.0)$ & $(2,2.10,389.6,423.2)$ & $(64,2.70,389.6,502.7)$ \\
\hline 75 & $(5,2.37,483.9,610.9)$ & $(16,2.55,484.2,611.5)$ & $(89,2.80,484.1,647.7)$ & $(37,2.66,484.2,602.6)$ & $(18,2.54,484.1,567.3)$ \\
\hline 80 & $(37,2.65,347.3,493.0)$ & $(69,2.72,347.6,481.5)$ & $(60,2.68,347.2,457.7)$ & $(33,2.58,347.2,434.9)$ & $(41,2.61,347.2,435.7)$ \\
\hline 85 & $(25,2.63,435.3,599.6)$ & $(19,2.56,435.5,553.9)$ & $(29,2.61,435.5,543.6)$ & $(62,2.72,435.1,560.6)$ & $(73,2.74,435.5,560.4)$ \\
\hline 90 & $(48,2.68,325.6,468.0)$ & $(53,2.67,325.9,443.3)$ & $(41,2.61,325.5,419.0)$ & $(56,2.65,325.8,421.7)$ & $(2,2.06,325.9,352.0)$ \\
\hline 95 & $(47,2.72,410.4,584.2)$ & $(33,2.64,410.8,539.2)$ & $(31,2.61,410.4,512.6)$ & $(37,2.63,410.3,512.2)$ & $(49,2.67,410.4,514.2)$ \\
\hline 100 & $(57,2.70,317.0,460.3)$ & $(20,2.51,317.1,406.2)$ & $(24,2.52,317.0,394.6)$ & $(39,2.59,317.0,399.5)$ & $(26,2.52,317.0,388.9)$ \\
\hline
\end{tabular}




\begin{tabular}{|c|c|c|c|c|c|}
\hline \multirow[b]{2}{*}{$c_{0}$} & \multicolumn{5}{|c|}{$\left(H^{\prime}{ }^{\prime}, K^{\prime}{ }^{\prime}, A R L_{0}, S D R L_{0}\right)$} \\
\hline & $m=10$ & $m=20$ & $m=50$ & $m=100$ & $m=200$ \\
\hline 5 & $(3,2.22,793.4,1522.9)$ & $(30,2.54,793.7,2538.1)$ & $(11,2.44,793.6,1602.1)$ & $(12,2.51,793.6,1502.5)$ & $(36,2.75,793.6,1096.6)$ \\
\hline 10 & $(61,2.70,517.3,948.4)$ & $(11,2.43,516.5,843.7)$ & $(56,2.70,517.0,879.2)$ & $(69,2.76,517.2,851.8)$ & $(86,2.82,517.7,789.4)$ \\
\hline 15 & $(62,2.57,210.8,358.4)$ & $(47,2.52,211.0,340.3)$ & $(72,2.59,211.1,317.5)$ & $(6,2.16,211.0,253.5)$ & $(40,2.51,210.9,295.7)$ \\
\hline 20 & $(25,2.59,442.3,683.8)$ & $(77,2.75,442.1,697.3)$ & $(60,2.71,442.4,647.8)$ & $(42,2.66,442.4,603.2)$ & $(45,2.68,442.9,571.3)$ \\
\hline 25 & $(30,2.58,344.5,526.0)$ & $(57,2.67,344.4,508.6)$ & $(57,2.66,344.3,480.3)$ & $(72,2.69,344.3,482.3)$ & $(87,2.72,344.3,480.3)$ \\
\hline 30 & $(39,2.62,313.0,473.6)$ & $(84,2.71,312.7,471.3)$ & $(64,2.66,312.7,441.6)$ & $(75,2.68,312.9,433.3)$ & $(40,2.58,312.9,409.6)$ \\
\hline 35 & $(73,2.71,310.9,483.2)$ & $(13,2.42,311.0,405.9)$ & $(73,2.68,310.9,435.1)$ & $(39,2.58,310.7,406.4)$ & $(30,2.54,310.8,390.0)$ \\
\hline 40 & $(57,2.69,327.0,494.0)$ & $(70,2.70,326.8,472.8)$ & $(37,2.59,327.0,428.6)$ & $(11,2.38,327.0,392.1)$ & $(65,2.67,327.1,429.8)$ \\
\hline 45 & $(70,2.74,357.1,541.7)$ & $(34,2.61,357.1,488.4)$ & $(73,2.71,357.1,488.6)$ & $(92,2.74,357.1,479.3)$ & $(4,2.21,357.2,400.4)$ \\
\hline 50 & $(68,2.76,400.7,602.3)$ & $(85,2.77,400.6,576.4)$ & $(88,2.76,400.6,551.6)$ & $(98,2.77,400.5,542.6)$ & $(20,2.52,400.9,484.1)$ \\
\hline 55 & $(30,2.66,457.8,649.4)$ & $(71,2.77,457.7,647.7)$ & $(91,2.79,457.9,625.0)$ & $(55,2.71,458.1,594.5)$ & $(56,2.71,458.2,586.6)$ \\
\hline 60 & $(45,2.63,274.0,399.6)$ & $(12,2.39,274.0,346.8)$ & $(55,2.62,274.1,365.5)$ & $(39,2.56,274.2,351.5)$ & $(52,2.60,274.0,356.4)$ \\
\hline 65 & $(81,2.75,327.1,489.0)$ & $(3,2.17,327.2,380.8)$ & $(61,2.67,327.1,436.5)$ & $(84,2.71,326.9,434.1)$ & $(44,2.61,327.2,415.1)$ \\
\hline 70 & $(51,2.72,393.0,569.6)$ & $(43,2.67,392.8,530.3)$ & $(19,2.52,393.0,483.2)$ & $(39,2.63,392.9,494.3)$ & $(52,2.67,393.0,498.6)$ \\
\hline 75 & $(56,2.77,474.3,689.0)$ & $(4,2.30,474.4,554.4)$ & $(34,2.65,474.3,598.2)$ & $(9,2.42,474.2,543.1)$ & $(13,2.48,474.2,549.0)$ \\
\hline 80 & $(85,2.76,321.8,479.1)$ & $(58,2.68,322.1,443.3)$ & $(21,2.50,321.7,398.3)$ & $(30,2.55,321.7,401.2)$ & $(29,2.54,321.8,398.8)$ \\
\hline 85 & $(36,2.67,394.6,556.9)$ & $(48,2.69,394.6,532.9)$ & $(27,2.58,394.6,491.7)$ & $(47,2.66,394.9,501.4)$ & $(59,2.69,394.7,503.2)$ \\
\hline 90 & $(87,2.84,484.7,718.2)$ & $(40,2.70,485.0,643.5)$ & $(72,2.77,484.8,635.8)$ & $(11,2.46,485.0,559.6)$ & $(73,2.76,484.9,621.2)$ \\
\hline 95 & $(47,2.69,349.3,500.0)$ & $(15,2.48,349.3,438.8)$ & $(15,2.46,349.2,420.0)$ & $(48,2.64,349.3,445.8)$ & $(69,2.69,349.1,451.6)$ \\
\hline 100 & $(86,2.82,432.8,636.8)$ & $(33,2.65,432.6,566.3)$ & $(59,2.72,432.6,562.4)$ & $(24,2.57,432.4,523.9)$ & $(60,2.71,432.6,548.8)$ \\
\hline
\end{tabular}




\begin{tabular}{|c|c|c|c|c|c|}
\hline \multirow[b]{2}{*}{$c_{0}$} & \multicolumn{5}{|c|}{$\left(H^{\prime}{ }^{\prime}, K_{c}^{\prime}, A R L_{0}, S D R L_{0}\right)$} \\
\hline & $m=10$ & $m=20$ & $m=50$ & $m=100$ & $m=200$ \\
\hline 5 & $(67,2.40,153.1,333.4)$ & $(15,2.23,153.0,264.8)$ & $(68,2.42,153.0,311.3)$ & $(59,2.44,153.1,281.2)$ & $(88,2.56,153.1,283.2)$ \\
\hline 10 & $(14,2.44,427.8,772.0)$ & $(35,2.59,427.6,723.5)$ & $(56,2.66,427.7,736.5)$ & $(82,2.75,427.8,712.2)$ & $(10,2.40,427.7,564.8)$ \\
\hline 15 & $(77,2.76,502.5,897.6)$ & $(92,2.78,502.6,893.5)$ & $(99,2.80,502.5,839.8)$ & $(75,2.77,502.4,765.8)$ & $(40,2.66,502.8,646.0)$ \\
\hline 20 & $(59,2.62,257.2,421.4)$ & $(74,2.64,257.4,403.9)$ & $(62,2.61,257.3,374.8)$ & $(53,2.58,257.4,367.6)$ & $(11,2.31,257.3,311.6)$ \\
\hline 25 & $(3,2.26,493.5,642.7)$ & $(4,2.29,493.2,614.5)$ & $(42,2.68,493.3,676.6)$ & $(24,2.59,493.4,625.4)$ & $(37,2.65,493.5,631.3)$ \\
\hline 30 & $(77,2.75,378.7,598.3)$ & $(1,1.97,378.5,429.5)$ & $(98,2.76,378.6,543.8)$ & $(80,2.73,378.7,519.4)$ & $(8,2.35,378.8,441.4)$ \\
\hline 35 & $(89,2.75,330.3,515.8)$ & $(36,2.60,330.3,459.1)$ & $(16,2.45,330.2,418.4)$ & $(53,2.64,330.2,443.9)$ & $(77,2.70,330.1,435.5)$ \\
\hline 40 & $(15,2.47,312.7,434.0)$ & $(12,2.41,312.7,401.4)$ & $(8,2.32,312.7,376.7)$ & $(7,2.29,312.6,366.3)$ & $(61,2.65,312.6,412.9)$ \\
\hline 45 & $(42,2.64,312.9,460.9)$ & $(9,2.36,313.0,391.3)$ & $(6,2.27,313.0,368.0)$ & $(64,2.66,312.7,416.2)$ & $(79,2.69,312.8,414.5)$ \\
\hline 50 & $(35,2.62,325.5,472.6)$ & $(18,2.49,325.5,424.1)$ & $(62,2.67,325.5,439.5)$ & $(28,2.54,325.7,408.4)$ & $(58,2.65,325.5,425.1)$ \\
\hline 55 & $(46,2.68,348.5,509.0)$ & $(87,2.75,348.6,497.6)$ & $(28,2.56,348.5,443.5)$ & $(18,2.48,348.4,421.5)$ & $(11,2.39,348.6,410.9)$ \\
\hline 60 & $(45,2.69,382.0,555.8)$ & $(67,2.73,381.8,535.3)$ & $(1,1.96,381.7,413.0)$ & $(36,2.61,381.7,483.3)$ & $(93,2.75,381.8,503.6)$ \\
\hline 65 & $(32,2.66,425.2,602.8)$ & $(11,2.46,425.2,527.0)$ & $(57,2.71,425.5,557.6)$ & $(4,2.25,425.5,476.1)$ & $(75,2.74,425.1,552.3)$ \\
\hline 70 & $(53,2.76,479.5,700.2)$ & $(86,2.81,480.4,677.0)$ & $(97,2.81,480.5,650.5)$ & $(72,2.76,479.9,625.8)$ & $(28,2.61,480.4,581.3)$ \\
\hline 75 & $(37,2.62,297.1,423.6)$ & $(74,2.70,297.2,415.4)$ & $(56,2.64,297.1,391.9)$ & $(89,2.70,297.2,395.1)$ & $(41,2.58,297.0,375.9)$ \\
\hline 80 & $(19,2.54,346.2,472.1)$ & $(36,2.62,346.1,461.3)$ & $(5,2.26,346.0,395.7)$ & $(31,2.57,346.2,430.2)$ & $(25,2.53,346.1,423.4)$ \\
\hline 85 & $(37,2.68,405.8,573.4)$ & $(85,2.78,405.7,566.9)$ & $(60,2.71,405.7,530.6)$ & $(63,2.71,405.7,522.7)$ & $(57,2.69,405.8,515.6)$ \\
\hline 90 & $(5,2.37,478.3,599.0)$ & $(97,2.83,478.4,667.9)$ & $(9,2.43,478.1,555.9)$ & $(19,2.55,478.4,571.0)$ & $(41,2.67,478.2,590.0)$ \\
\hline 95 & $(51,2.69,326.9,470.7)$ & $(69,2.71,326.9,450.9)$ & $(98,2.74,326.8,441.2)$ & $(78,2.70,326.7,428.8)$ & $(3,2.14,326.7,358.2)$ \\
\hline 100 & $(30,2.64,390.1,540.6)$ & $(95,2.79,390.5,545.1)$ & $(67,2.72,390.7,513.7)$ & $(71,2.72,390.5,507.3)$ & $(26,2.56,390.7,473.7)$ \\
\hline
\end{tabular}




\begin{tabular}{|c|c|c|c|c|c|c|}
\hline$p_{0}$ & $m=10$ & $m=20$ & $m=50$ & $m=100$ & $m=200$ & $m=\infty$ \\
\hline & \multicolumn{6}{|c|}{$n=25$} \\
\hline 0.01 & $(17028.6,3188226.0)$ & $(2309.0,38950.9)$ & $(738.1,4301.8)$ & $(735.7,826.3)$ & $(760.2,799.2)$ & $(763.4,799.4)$ \\
\hline 0.02 & $(10228.7,2197057.6)$ & $(1975.8,21891.2)$ & $(1322.5,2663.6)$ & $(1206.7,2328.2)$ & $(1066.1,2212.2)$ & $(66.6,75.3)$ \\
\hline 0.05 & $(5881.1,441950.1)$ & $(1764.5,12937.7)$ & $(882.5,3020.2)$ & $(518.8,1361.9)$ & $(442.0,548.9)$ & $(437.7,464.2)$ \\
\hline 0.10 & $(3717.6,19936.6)$ & $(2025.3,9211.2)$ & $(1063.0,2702.0)$ & $(725.1,1802.7)$ & $(516.8,962.3)$ & $(455.8,483.0)$ \\
\hline 0.15 & $(459.3,684.0)$ & $(471.3,654.0)$ & $(477.0,632.0)$ & $(472.3,615.1)$ & $(456.3,592.9)$ & $(280.7,301.3)$ \\
\hline \multirow[t]{2}{*}{0.20} & $(464.8,752.8)$ & $(512.7,803.8)$ & $(668.0,956.8)$ & $(787.7,1040.8)$ & $(924.0,1111.1)$ & $(1134.0,1178.5)$ \\
\hline & \multicolumn{6}{|c|}{$n=50$} \\
\hline 0.01 & $(12150.6,3182832.7)$ & $(1779.1,22060.4)$ & $(1217.6,2425.4)$ & $(1112.1,2140.4)$ & $(1081.0,2115.8)$ & $(65.4,74.1)$ \\
\hline 0.02 & $(8406.4,731146.3)$ & $(1880.6,13849.2)$ & $(981.5,2121.4)$ & $(1043.5,1507.6)$ & $(1108.2,1533.7)$ & $(1599.7,1653.2)$ \\
\hline 0.05 & $(3444.5,14337.2)$ & $(1785.6,7503.6)$ & $(1061.7,2350.3)$ & $(776.3,1756.2)$ & $(529.6,1181.0)$ & $(357.1,380.8)$ \\
\hline 0.10 & $(535.0,1015.3)$ & $(630.8,1121.4)$ & $(637.2,997.8)$ & $(588.4,764.3)$ & $(571.6,625.4)$ & $(575.7,606.6)$ \\
\hline 0.15 & $(383.5,563.1)$ & $(416.1,560.7)$ & $(404.6,513.9)$ & $(373.0,471.3)$ & $(331.2,415.2)$ & $(261.1,281.0)$ \\
\hline \multirow[t]{2}{*}{0.20} & $(337.8,443.1)$ & $(366.9,449.0)$ & $(374.6,432.5)$ & $(362.6,416.7)$ & $(342.1,399.8)$ & $(210.9,228.5)$ \\
\hline & \multicolumn{6}{|c|}{$n=75$} \\
\hline 0.01 & $(9173.4,1886763.6)$ & $(1748.3,16414.7)$ & $(722.4,2927.7)$ & $(405.1,1358.7)$ & $(327.2,430.5)$ & $(324.2,346.6)$ \\
\hline 0.02 & $(6748.1,432961.3)$ & $(1598.2,10555.4)$ & $(954.5,2080.0)$ & $(964.7,1490.3)$ & $(1015.7,1515.2)$ & $(1666.2,1720.7)$ \\
\hline 0.05 & $(714.5,1521.3)$ & $(707.6,1471.1)$ & $(754.2,1506.4)$ & $(717.9,1402.6)$ & $(597.2,1092.1)$ & $(449.7,476.6)$ \\
\hline 0.10 & $(377.3,536.2)$ & $(392.8,510.9)$ & $(380.8,451.0)$ & $(367.9,429.6)$ & $(348.7,414.5)$ & $(201.6,218.8)$ \\
\hline 0.15 & $(345.8,470.2)$ & $(368.8,457.8)$ & $(387.5,444.3)$ & $(401.8,442.2)$ & $(412.2,448.2)$ & $(411.5,437.2)$ \\
\hline \multirow[t]{2}{*}{0.20} & $(308.4,402.6)$ & $(352.3,439.2)$ & $(396.3,480.0)$ & $(431.2,515.5)$ & $(478.9,556.5)$ & $(597.7,629.2)$ \\
\hline & \multicolumn{6}{|c|}{$n=100$} \\
\hline 0.01 & $(7363.7,1090099.2)$ & $(1741.4,14829.8)$ & $(917.8,1948.2)$ & $(975.3,1409.7)$ & $(1071.2,1447.5)$ & $(1494.8,1546.3)$ \\
\hline 0.02 & $(5976.9,127400.8)$ & $(1646.1,10120.0)$ & $(972.3,2058.2)$ & $(903.0,1616.5)$ & $(795.2,1509.6)$ & $(198.6,215.5)$ \\
\hline 0.05 & $(580.4,1034.2)$ & $(627.2,1042.3)$ & $(572.7,886.7)$ & $(508.5,707.3)$ & $(458.3,542.8)$ & $(437.2,463.7)$ \\
\hline 0.10 & $(346.6,467.2)$ & $(389.0,496.4)$ & $(434.3,535.5)$ & $(484.5,578.1)$ & $(539.1,615.6)$ & $(627.3,659.6)$ \\
\hline 0.15 & $(311.4,408.4)$ & $(353.0,435.7)$ & $(384.0,451.9)$ & $(407.1,467.2)$ & $(422.6,466.5)$ & $(432.2,458.6)$ \\
\hline 0.20 & $(301.3,376.6)$ & $(340.0,402.2)$ & $(374.0,428.4)$ & $(398.1,450.5)$ & $(427.0,473.9)$ & $(478.4,506.3)$ \\
\hline 0.10 & $(627.3,659.6)$ & $(627.3,659.6)$ & $(627.3,659.6)$ & $(627.3,659.6)$ & $(627.3,659.6)$ & $(627.3,659.6)$ \\
\hline 0.15 & $(432.2,458.6)$ & $(432.2,458.6)$ & $(432.2,458.6)$ & $(432.2,458.6)$ & $(432.2,458.6)$ & $(432.2,458.6)$ \\
\hline 0.20 & $(478.4,506.3)$ & $(478.4,506.3)$ & $(478.4,506.3)$ & $(478.4,506.3)$ & $(478.4,506.3)$ & $(478.4,506.3)$ \\
\hline
\end{tabular}




\begin{tabular}{|c|c|c|c|c|c|c|}
\hline$p_{0}$ & $m=10$ & $m=20$ & $m=50$ & $m=100$ & $m=200$ & $m=\infty$ \\
\hline & \multicolumn{6}{|c|}{$n=25$} \\
\hline 0.01 & $(19682.6,5739672.4)$ & $(1429.1,39697.7)$ & $(401.0,3678.4)$ & $(234.7,591.1)$ & $(232.5,265.3)$ & $(232.5,264.7)$ \\
\hline 0.02 & $(11169.4,3839976.4)$ & $(1394.5,21014.3)$ & $(609.2,1928.9)$ & $(629.5,868.5)$ & $(708.3,890.2)$ & $(847.5,915.8)$ \\
\hline 0.05 & $(6045.1,1204194.0)$ & $(1374.6,13474.5)$ & $(722.6,1938.6)$ & $(531.1,1492.2)$ & $(335.3,1083.7)$ & $(136.1,159.1)$ \\
\hline 0.10 & $(5461.1,58554.2)$ & $(1805.8,12242.4)$ & $(892.7,2079.6)$ & $(824.7,1403.0)$ & $(815.2,1387.3)$ & $(141.5,165.1)$ \\
\hline 0.15 & $(867.7,1680.6)$ & $(999.3,1798.0)$ & $(996.7,1787.8)$ & $(929.4,1720.5)$ & $(819.4,1601.5)$ & $(237.7,270.3)$ \\
\hline \multirow[t]{2}{*}{0.20} & $(630.2,1168.6)$ & $(630.0,1116.5)$ & $(556.2,958.2)$ & $(464.6,763.3)$ & $(381.5,537.9)$ & $(341.5,382.0)$ \\
\hline & \multicolumn{6}{|c|}{$n=50$} \\
\hline 0.01 & $(9910.7,4834585.4)$ & $(1334.6,24824.9)$ & $(559.5,1670.8)$ & $(613.6,814.2)$ & $(670.1,825.6)$ & $(779.9,845.1)$ \\
\hline 0.02 & $(7817.2,1684253.9)$ & $(1483.6,14069.5)$ & $(510.2,1865.0)$ & $(446.0,663.2)$ & $(467.5,525.9)$ & $(477.7,527.1)$ \\
\hline 0.05 & $(5455.1,129198.0)$ & $(1374.9,9720.5)$ & $(742.4,1775.7)$ & $(697.4,1046.4)$ & $(747.4,1038.5)$ & $(1065.3,1142.8)$ \\
\hline 0.10 & $(588.1,1131.9)$ & $(491.7,850.4)$ & $(438.5,633.4)$ & $(408.0,577.0)$ & $(384.2,556.7)$ & $(177.1,204.3)$ \\
\hline 0.15 & $(431.4,763.1)$ & $(447.4,689.4)$ & $(494.9,679.3)$ & $(530.6,660.8)$ & $(555.3,635.3)$ & $(580.3,635.5)$ \\
\hline 0.20 & $(373.9,598.1)$ & $(424.7,641.1)$ & $(447.2,630.9)$ & $(433.5,574.1)$ & $(400.3,486.4)$ & $(375.6,418.5)$ \\
\hline 0.01 & $(7768.3,2323204.2)$ & $(1315.1,16163.6)$ & $(701.5,1962.5)$ & $(434.9,1423.7)$ & $(223.2,878.6)$ & $(102.2,121.3)$ \\
\hline 0.02 & $(5939.7,1427754.2)$ & $(1311.8,12221.6)$ & $(580.2,1803.7)$ & $(474.0,763.5)$ & $(484.8,547.8)$ & $(497.1,547.6)$ \\
\hline 0.05 & $(2306.2,12557.0)$ & $(1319.2,5081.3)$ & $(791.5,1881.5)$ & $(703.4,1050.5)$ & $(749.5,965.0)$ & $(960.3,1033.6)$ \\
\hline 0.10 & $(475.8,838.9)$ & $(528.2,881.1)$ & $(516.1,800.7)$ & $(477.5,682.6)$ & $(430.1,538.3)$ & $(405.0,449.8)$ \\
\hline 0.15 & (332.9 ARL, 490.5) & $(381.8,524.0)$ & $(398.0,516.0)$ & $(396.5,498.6)$ & $(398.0,486.9)$ & $(321.7,360.9)$ \\
\hline \multirow[t]{2}{*}{0.20} & $(319.5,455.1)$ & $(358.0,475.3)$ & $(375.1,469.9)$ & $(389.2,487.5)$ & $(407.7,517.4)$ & $(817.7,884.7)$ \\
\hline & \multicolumn{6}{|c|}{$n=100$} \\
\hline 0.01 & $(6575.4,1791230.0)$ & $(1328.9,12824.0)$ & $(530.1,1953.0)$ & $(425.5,655.9)$ & $(438.9,493.8)$ & $(447.0,494.5)$ \\
\hline 0.02 & $(5548.7,636777.1)$ & $(1333.2,10874.2)$ & $(618.7,1724.3)$ & $(543.2,816.8)$ & $(578.8,672.9)$ & $(624.1,681.6)$ \\
\hline 0.05 & $(583.7,1046.1)$ & $(514.9,859.4)$ & $(508.0,772.8)$ & $(548.6,818.6)$ & $(619.5,902.8)$ & $(1123.3,1203.2)$ \\
\hline 0.10 & $(374.0,571.9)$ & $(395.3,552.6)$ & $(380.1,477.4)$ & $(371.6,443.6)$ & $(362.7,432.8)$ & $(192.3,221.0)$ \\
\hline 0.15 & $(323.0,446.3)$ & $(358.8,468.2)$ & $(396.3,494.0)$ & $(430.0,523.4)$ & $(471.3,555.4)$ & $(545.5,598.8)$ \\
\hline 0.20 & $(301.6,410.7)$ & $(335.6,429.8)$ & $(382.0,468.2)$ & $(407.1,491.7)$ & $(442.9,522.7)$ & $(523.4,575.4)$ \\
\hline
\end{tabular}




\begin{tabular}{|c|c|c|c|c|c|c|}
\hline$p_{0}$ & $m=10$ & $m=20$ & $m=50$ & $m=100$ & $m=200$ & $m=\infty$ \\
\hline & & & $n=25$ & & & \\
\hline 0.01 & $(21078.6,43052425.3)$ & $(1198.1,51986.2)$ & $(227.4,1482.6)$ & $(86.5,637.0)$ & $(56.2,145.7)$ & $(54.9,71.5)$ \\
\hline 0.02 & $(13562.5,6999996.8)$ & $(1113.7,25969.2)$ & $(231.3,1425.1)$ & $(160.5,400.3)$ & $(161.1,209.4)$ & $(162.2,208.9)$ \\
\hline 0.05 & $(6655.9,3575578.3)$ & $(1060.5,15197.8)$ & $(371.9,1303.5)$ & $(347.9,562.8)$ & $(383.6,553.4)$ & $(486.7,591.7)$ \\
\hline 0.10 & $(6352.5,445371.7)$ & $(1330.2,13596.3)$ & $(521.0,1650.3)$ & $(349.9,879.2)$ & $(296.6,433.9)$ & $(292.5,366.0)$ \\
\hline 0.15 & $(3566.3,20544.7)$ & $(1473.3,8798.5)$ & $(712.0,2063.0)$ & $(494.6,1181.5)$ & $(408.7,602.4)$ & $(400.0,491.9)$ \\
\hline 0.20 & $(496.5,821.9)$ & $(526.8,811.8)$ & $\begin{array}{r}(600.3,848.6) \\
n=50\end{array}$ & $(665.7,881.1)$ & $(726.4,906.5)$ & $(781.5,924.9)$ \\
\hline 0.01 & $(10143.5,17237428.1)$ & $(930.7,18388.9)$ & $(213.8,1201.8)$ & $(150.7,350.4)$ & $(150.1,195.5)$ & $(150.8,194.8)$ \\
\hline 0.02 & $(7482.7,6985147.7)$ & $(770.6,14014.0)$ & $(339.2,1164.3)$ & $(186.2,671.2)$ & $(115.8,318.9)$ & $(98.9,129.3)$ \\
\hline 0.05 & $(5633.5,1380226.6)$ & $(1094.4,10012.7)$ & $(449.0,1269.5)$ & $(313.5,809.8)$ & $(223.8,440.6)$ & $(198.6,253.5)$ \\
\hline 0.10 & $(1485.8,5869.1)$ & $(986.5,3645.6)$ & $(642.6,1604.2)$ & $(495.3,1063.4)$ & $(371.1,708.1)$ & $(299.4,374.2)$ \\
\hline 0.15 & $(485.9,864.1)$ & $(476.1,780.7)$ & $(471.7,725.3)$ & $(516.9,757.3)$ & $(587.2,814.7)$ & $(774.3,916.8)$ \\
\hline 0.20 & $(401.2,746.8)$ & $(448.8,795.8)$ & $\begin{array}{r}(479.0,790.9) \\
n=75\end{array}$ & $(473.4,717.3)$ & $(451.0,601.3)$ & $(442.4,540.9)$ \\
\hline 0.01 & $(7292.9,6297830.9)$ & $(783.8,16722.7)$ & $(284.2,999.2)$ & $(282.0,518.2)$ & $(295.7,524.3)$ & $(518.9,628.6)$ \\
\hline 0.02 & $(5055.6,5296151.5)$ & $(865.5,10507.5)$ & $(364.7,1074.3)$ & $(262.7,737.8)$ & $(163.1,472.5)$ & $(102.3,133.7)$ \\
\hline 0.05 & $(5313.4,99607.5)$ & $(1145.8,9487.5)$ & $(522.9,1327.9)$ & $(408.0,866.0)$ & $(307.0,668.1)$ & $(181.1,232.2)$ \\
\hline 0.10 & $(487.2,951.0)$ & $(485.9,918.4)$ & $(489.0,893.8)$ & $(473.8,762.3)$ & $(464.1,623.5)$ & $(484.7,589.4)$ \\
\hline 0.15 & $(396.2,708.7)$ & $(415.6,676.6)$ & $(408.5,613.7)$ & $(389.1,549.9)$ & $(379.3,502.4)$ & $(342.8,425.2)$ \\
\hline 0.20 & $(338.0,537.9)$ & $(376.3,566.3)$ & $\begin{array}{r}(403.5,582.0) \\
n=100\end{array}$ & $(428.6,608.1)$ & $(472.2,657.2)$ & $(698.5,831.9)$ \\
\hline 0.01 & $(6050.2,7913163.8)$ & $(819.5,11914.4)$ & $(306.7,1025.9)$ & $(188.8,657.2)$ & $(111.9,311.0)$ & $(93.6,122.4)$ \\
\hline 0.02 & $(6045.4,3519398.5)$ & $(838.0,10561.6)$ & $(378.3,1093.5)$ & $(278.7,717.8)$ & $(191.0,492.2)$ & $(124.3,161.6)$ \\
\hline 0.05 & $(1973.0,11694.0)$ & $(1021.5,4485.2)$ & $(547.1,1346.2)$ & $(447.6,876.0)$ & $(365.0,723.3)$ & $(208.2,265.2)$ \\
\hline 0.10 & $(431.3,817.1)$ & $(440.4,784.7)$ & $(398.4,627.9)$ & $(373.5,542.6)$ & $(356.8,515.4)$ & $(193.8,247.7)$ \\
\hline 0.15 & $(341.7,549.6)$ & $(369.1,564.8)$ & $(404.1,595.6)$ & $(417.4,584.9)$ & $(424.8,559.0)$ & $(433.2,530.3)$ \\
\hline 0.20 & $(310.9,490.5)$ & $(351.0,522.5)$ & $(388.7,555.2)$ & $(398.9,553.0)$ & $(392.6,523.4)$ & $(364.3,450.3)$ \\
\hline
\end{tabular}

\begin{tabular}{|c|c|c|c|c|}
\hline$p_{0}$ & $n=25$ & $n=50$ & $n=75$ & $n=100$ \\
\hline & \multicolumn{4}{|c|}{$\left(H_{n p}=2, K_{n p}=2.085\right)$} \\
\hline 0.01 & 50 & $>10,000$ & 160 & 1920 \\
\hline 0.02 & $>10,000$ & 2530 & 20 & 5770 \\
\hline 0.05 & 150 & 490 & 480 & 200 \\
\hline 0.10 & 270 & 80 & 5680 & 480 \\
\hline 0.15 & 5320 & 670 & 60 & 110 \\
\hline \multirow[t]{2}{*}{0.20} & 590 & 3510 & 740 & 390 \\
\hline & \multicolumn{4}{|c|}{$\left(H_{n p}=7, K_{n p}=2.322\right)$} \\
\hline 0.01 & 80 & 500 & 560 & 60 \\
\hline 0.02 & 570 & 60 & 60 & 50 \\
\hline 0.05 & 740 & 30 & 1200 & 6790 \\
\hline 0.10 & $>10,000$ & 7640 & 220 & $>10,000$ \\
\hline 0.15 & 3960 & 180 & 10 & 480 \\
\hline \multirow[t]{2}{*}{0.20} & 290 & 10 & $>10,000$ & 570 \\
\hline & \multicolumn{4}{|c|}{$\left(H_{n p}=47, K_{n p}=2.639\right)$} \\
\hline 0.01 & 190 & 80 & $>10,000$ & 300 \\
\hline 0.02 & 90 & 270 & 500 & 520 \\
\hline 0.05 & 850 & 270 & 770 & 1020 \\
\hline 0.10 & 160 & 370 & 10 & 9150 \\
\hline 0.15 & 170 & 1050 & 1070 & 80 \\
\hline 0.20 & 260 & 20 & 2010 & 20 \\
\hline
\end{tabular}




\begin{tabular}{|c|c|c|c|c|c|}
\hline \multirow[b]{2}{*}{$p_{0}$} & \multicolumn{5}{|c|}{$\left(H_{n p}^{\prime}, K_{n p}^{\prime} A R L_{0}, S D R L_{0}\right)$} \\
\hline & $m=10$ & $m=20$ & $m=50$ & $m=100$ & $m=200$ \\
\hline & \multicolumn{5}{|c|}{$n=25$} \\
\hline 0.01 & $(23,2.04,760.7,266840.9)$ & $(76,2.72,763.2,32209.3)$ & $(50,2.98,761.8,3681.0)$ & $(7,2.75,762.3,6352.4)$ & $(2,2.39,763.4,813.3)$ \\
\hline 0.02 & $(84,1.73,66.6,3154.5)$ & $(96,2.14,66.6,614.8)$ & $(4,1.57,66.6,305.6)$ & $(54,2.10,66.6,139.3)$ & $(2,1.37,66.6,75.3)$ \\
\hline 0.05 & $(14,1.93,437.2,11761.1)$ & $(45,2.44,437.6,4159.1)$ & $(73,2.81,437.2,1853.8)$ & $(16,2.43,437.5,959.3)$ & $(53,2.88,437.8,588.5)$ \\
\hline 0.10 & $(78,2.26,456.0,5233.8)$ & $(20,2.25,455.9,2796.4)$ & $(12,2.27,455.8,995.6)$ & $(97,2.81,455.8,1214.9)$ & $(2,1.96,455.9,499.7)$ \\
\hline 0.15 & $(78,2.40,280.5,1030.3)$ & $(95,2.44,280.8,919.7)$ & $(56,2.46,280.7,699.1)$ & $(51,2.46,280.6,443.2)$ & $(75,2.63,280.7,404.3)$ \\
\hline 0.20 & $(5,2.37,1135.1,2146.9)$ & \multicolumn{3}{|c|}{$n=50$} & $(40,2.80,1133.6,2535.8)$ \\
\hline 0.01 & $(71,1.67,65.4,2093.2)$ & $(86,2.07,65.4,472.4)$ & $(94,2.28,65.4,142.5)$ & $(2,1.32,65.4,75.8)$ & $(2,1.36,65.4,74.1)$ \\
\hline 0.02 & $(35,2.32,1601.3,336911.3)$ & $(27,2.68,1599.0,26314.3)$ & $(18,2.83,1597.7,5407.4)$ & $(4,2.61,1599.3,6253.8)$ & $(2,2.45,1599.5,1772.6)$ \\
\hline 0.05 & $(62,2.20,357.2,2886.6)$ & $(7,1.99,357.2,1492.5)$ & $(4,1.99,357.2,854.9)$ & $(31,2.59,357.1,871.8)$ & $(24,2.59,357.2,559.4)$ \\
\hline 0.10 & $(14,2.46,575.6,948.7)$ & $(31,2.52,576.0,1424.8)$ & $(5,2.31,575.9,835.1)$ & $(27,2.57,576.3,1155.9)$ & $(74,2.77,575.8,1108.8)$ \\
\hline 0.15 & $(19,2.38,261.2,493.9)$ & $(85,2.62,261.0,450.4)$ & $(28,2.44,261.1,424.8)$ & $(14,2.30,261.1,333.4)$ & $(20,2.43,261.3,376.9)$ \\
\hline 0.20 & \multicolumn{5}{|c|}{$n=75$} \\
\hline 0.01 & $(65,2.15,324.2,73701.8)$ & $(77,2.56,324.1,4949.7)$ & $(33,2.53,323.8,844.8)$ & $(59,2.78,324.2,529.9)$ & $(83,3.00,324.2,433.2)$ \\
\hline 0.02 & $(14,2.18,1666.6,105139.7)$ & $(53,2.80,1666.1,27956.6)$ & $(22,2.86,1666.7,5734.4)$ & $(10,2.72,1668.8,4015.0)$ & $(2,2.41,1666.5,1881.3)$ \\
\hline 0.05 & $(26,2.31,449.6,1234.0)$ & $(77,2.53,449.4,2627.0)$ & $(24,2.47,449.4,1139.0)$ & $(38,2.62,449.7,968.7)$ & $(33,2.65,449.9,977.7)$ \\
\hline 0.10 & $(18,2.32,201.7,366.0)$ & $(81,2.56,201.8,341.3)$ & $(10,2.21,201.7,276.5)$ & $(96,2.57,201.6,303.3)$ & $(73,2.53,201.7,312.6)$ \\
\hline 0.15 & $(10,2.41,411.4,634.6)$ & $(15,2.46,411.7,622.2)$ & $(44,2.63,411.5,620.2)$ & $(94,2.74,411.3,650.2)$ & $(56,2.68,411.6,542.5)$ \\
\hline 0.20 & \multicolumn{5}{|c|}{$n=100$} \\
\hline 0.01 & $(3,1.84,1495.1,50275.0)$ & $(38,2.76,1495.1,29623.0)$ & $(32,2.97,1495.0,6107.5)$ & $(2,2.37,1497.0,2840.7)$ & $(25,2.97,1494.7,2920.7)$ \\
\hline 0.02 & $(17,1.87,198.5,941.4)$ & $(35,2.21,198.6,1256.6)$ & $(95,2.61,198.4,550.0)$ & $(38,2.52,198.6,504.3)$ & $(9,2.09,198.5,381.7)$ \\
\hline 0.05 & $(11,2.35,437.2,776.8)$ & $(85,2.58,437.2,1438.7)$ & $(61,2.63,437.3,1070.1)$ & $(89,2.75,437.2,781.9)$ & $(65,2.71,437.2,789.0)$ \\
\hline 0.10 & $(55,2.72,627.6,1173.6)$ & $(52,2.72,627.6,1072.7)$ & $(42,2.70,627.3,964.2)$ & $(92,2.81,627.2,1060.7)$ & $(97,2.85,627.4,987.7)$ \\
\hline 0.15 & $(97,2.77,432.1,776.0)$ & $(94,2.76,432.3,718.3)$ & $(33,2.60,432.1,585.8)$ & $(5,2.27,432.3,544.5)$ & $(96,2.78,432.3,607.3)$ \\
\hline 0.20 & $(54,2.74,478.4,759.1)$ & $(20,2.56,478.4,652.7)$ & $(8,2.39,478.5,606.8)$ & $(88,2.77,478.7,676.3)$ & $(51,2.70,478.2,642.3)$ \\
\hline
\end{tabular}




\begin{tabular}{|c|c|c|c|c|c|}
\hline \multirow[b]{2}{*}{$p_{0}$} & \multicolumn{5}{|c|}{$\left(H_{n p}^{\prime}, K_{n p}^{\prime}, A R L_{0}, S D R L_{0}\right)$} \\
\hline & $m=10$ & $m=20$ & $m=50$ & $m=100$ & $m=200$ \\
\hline & & & $n=25$ & & \\
\hline 0.01 & $(43,1.53,54.8,1754.5)$ & $(72,1.85,55.0,433.6)$ & $(38,1.94,54.9,102.8)$ & $(67,2.55,54.9,279.1)$ & $(47,2.41,54.9,72.1)$ \\
\hline 0.02 & $(40,1.85,162.4,14058.9)$ & $(28,2.14,162.2,1995.0)$ & $(75,2.64,161.9,927.7)$ & $(63,2.80,162.1,750.9)$ & $(46,2.57,162.2,211.1)$ \\
\hline 0.05 & $(86,2.26,486.6,89653.5)$ & $(11,2.17,486.6,3999.8)$ & $(33,2.62,485.8,1556.9)$ & $(69,2.96,486.6,1804.7)$ & $(47,2.90,486.6,675.3)$ \\
\hline 0.10 & $(1,1.76,292.5,573.8)$ & $(26,2.21,292.4,1630.0)$ & $(50,2.51,292.3,882.2)$ & $(48,2.58,292.5,589.7)$ & $(34,2.41,292.6,424.2)$ \\
\hline 0.15 & $(51,2.40,399.5,1497.2)$ & $(76,2.49,399.9,1267.7)$ & $(44,2.50,399.6,1086.5)$ & $(79,2.69,400.1,1025.4)$ & $(67,2.74,400.1,956.1)$ \\
\hline 0.20 & $(58,2.67,781.5,2386.6)$ & $(13,2.50,781.5,1345.9)$ & $\begin{array}{c}(41,2.66,781.5,1503.5) \\
n=50\end{array}$ & $(50,2.69,781.7,1621.7)$ & $(14,2.51,781.4,1308.4)$ \\
\hline 0.01 & $(38,1.83,150.8,18309.3)$ & $(71,2.32,150.6,2502.9)$ & $(48,2.53,150.7,730.1)$ & $(47,2.64,150.7,350.4)$ & $(58,2.96,150.8,581.6)$ \\
\hline 0.02 & $(33,1.75,99.0,1959.4)$ & $(34,2.01,98.9,634.6)$ & $(66,2.38,99.0,328.3)$ & $(44,2.35,98.9,165.7)$ & $(46,2.34,98.9,130.6)$ \\
\hline 0.05 & $(66,2.11,198.4,1474.4)$ & $(75,2.35,198.5,1220.2)$ & $(20,2.22,198.6,446.9)$ & $(68,2.60,198.7,474.7)$ & $(46,2.51,198.5,264.0)$ \\
\hline 0.10 & $(15,2.35,299.2,588.0)$ & $(13,2.33,299.4,522.6)$ & $(88,2.59,299.5,668.5)$ & $(81,2.63,299.4,634.9)$ & $(44,2.51,299.4,399.1)$ \\
\hline 0.15 & $(87,2.79,774.3,1604.2)$ & $(48,2.72,774.1,1549.1)$ & $(96,2.81,774.0,1501.4)$ & $(28,2.63,774.9,1114.5)$ & $(42,2.69,774.2,968.2)$ \\
\hline 0.20 & $(96,2.74,442.9,858.5)$ & $(69,2.69,442.4,782.5)$ & $\begin{array}{c}(6,2.29,442.3,609.1) \\
n=75\end{array}$ & $(91,2.75,442.5,706.0)$ & $(46,2.62,442.5,574.3)$ \\
\hline 0.01 & $(11,1.91,517.8,45742.1)$ & $(15,2.27,518.9,5161.2)$ & $(59,2.92,519.0,2684.7)$ & $(1,1.62,519.1,638.0)$ & $(46,3.00,519.2,661.2)$ \\
\hline 0.02 & $(33,1.80,102.4,1305.2)$ & $(96,2.25,102.2,572.6)$ & $(60,2.35,102.3,292.9)$ & $(56,2.44,102.3,228.4)$ & $(48,2.46,102.3,150.7)$ \\
\hline 0.05 & $(74,2.29,180.9,508.2)$ & $(67,2.33,181.1,708.5)$ & $(49,2.39,181.1,433.8)$ & $(34,2.34,181.1,289.4)$ & $(56,2.55,181.1,323.0)$ \\
\hline 0.10 & $(55,2.66,484.4,993.1)$ & $(8,2.34,484.5,796.2)$ & $(90,2.73,484.7,910.0)$ & $(83,2.75,484.5,884.4)$ & $(46,2.65,484.8,663.8)$ \\
\hline 0.15 & $(41,2.59,342.6,606.0)$ & $(79,2.68,342.7,565.2)$ & $(55,2.63,342.7,522.8)$ & $(24,2.47,342.7,501.5)$ & $(66,2.67,342.6,461.4)$ \\
\hline 0.20 & $(19,2.63,698.5,1080.9)$ & $(8,2.46,698.2,995.6)$ & $\begin{array}{c}(94,2.84,698.4,1056.4) \\
n=100\end{array}$ & $(2,2.20,698.3,825.4)$ & $(58,2.78,698.5,984.7)$ \\
\hline 0.01 & $(55,1.81,93.6,2715.0)$ & $(27,1.96,93.4,520.2)$ & $(65,2.37,93.6,299.3)$ & $(63,2.51,93.6,254.9)$ & $(49,2.54,93.5,157.0)$ \\
\hline 0.02 & $(73,2.01,124.2,1193.3)$ & $(85,2.29,124.2,754.3)$ & $(55,2.36,124.3,329.2)$ & $(3,1.63,124.3,154.5)$ & $(40,2.29,124.2,172.0)$ \\
\hline 0.05 & $(60,2.44,208.2,479.2)$ & $(32,2.41,208.1,322.7)$ & $(30,2.38,208.2,311.8)$ & $(67,2.53,208.1,407.7)$ & $(51,2.52,208.2,323.8)$ \\
\hline 0.10 & $(97,2.58,193.9,369.2)$ & $(7,2.17,193.8,288.9)$ & $(92,2.58,193.9,316.4)$ & $(78,2.57,193.8,303.9)$ & $(39,2.42,193.8,261.1)$ \\
\hline 0.15 & $(35,2.63,433.4,694.4)$ & $(28,2.59,433.1,625.7)$ & $(84,2.74,433.0,658.2)$ & $(90,2.76,433.3,627.6)$ & $(30,2.56,433.1,587.1)$ \\
\hline 0.20 & $(74,2.73,364.3,593.9)$ & $(21,2.52,364.2,505.7)$ & $(32,2.57,364.2,487.4)$ & $(53,2.64,364.2,510.2)$ & $(60,2.67,364.2,500.6)$ \\
\hline
\end{tabular}




\begin{tabular}{|c|c|c|c|c|c|}
\hline \multirow[b]{2}{*}{$p_{0}$} & \multicolumn{5}{|c|}{$\left(H_{n p}^{\prime}, K_{n p}^{\prime}, A R L_{0}, S D R L_{0}\right)$} \\
\hline & $m=10$ & $m=20$ & $m=50$ & $m=100$ & $m=200$ \\
\hline & \multicolumn{5}{|c|}{$n=25$} \\
\hline 0.01 & $(8,1.43,232.8,9332.6)$ & $(45,2.20,232.6,3360.3)$ & $(28,2.49,233.2,1801.3)$ & $(73,2.92,232.1,1260.0)$ & $(7,2.39,232.5,268.2)$ \\
\hline 0.02 & $(38,2.21,847.3,294492.6)$ & $(63,2.69,847.2,19408.6)$ & $(67,3.00,846.5,3699.2)$ & $(6,2.42,847.9,1064.1)$ & $(7,2.75,847.6,1037.0)$ \\
\hline 0.05 & $(27,1.78,136.1,1606.5)$ & $(67,2.26,136.1,1063.0)$ & $(2,1.47,136.0,295.0)$ & $(18,2.24,136.0,434.4)$ & $(1,1.46,136.1,362.1)$ \\
\hline 0.10 & $(96,2.11,141.4,648.8)$ & $(44,2.13,141.5,578.4)$ & $(26,2.16,141.5,335.0)$ & $(70,2.37,141.5,237.6)$ & $(7,1.96,141.5,169.4)$ \\
\hline 0.15 & $(97,2.40,237.1,856.9)$ & $(26,2.27,237.6,492.3)$ & $(85,2.50,237.5,619.3)$ & $(11,2.20,237.7,534.4)$ & $(85,2.53,237.6,323.0)$ \\
\hline 0.20 & \multicolumn{5}{|c|}{$n=50$} \\
\hline 0.01 & $(96,2.39,779.0,758660.5)$ & $(98,2.79,778.8,20972.0)$ & $(61,2.98,780.3,3301.3)$ & $(6,2.44,780.0,1029.7)$ & $(7,2.73,779.9,943.4)$ \\
\hline 0.02 & $(73,2.23,477.7,83669.3)$ & $(79,2.61,477.9,6232.2)$ & $(6,2.23,477.7,1555.2)$ & $(1,1.78,478.2,1403.0)$ & $(7,2.45,477.6,558.7)$ \\
\hline 0.05 & $(62,2.39,1065.5,48341.6)$ & $(38,2.60,1064.7,9668.9)$ & $(62,2.90,1065.4,3480.2)$ & $(20,2.73,1065.0,2641.4)$ & $(7,2.54,1065.6,1299.3)$ \\
\hline 0.10 & $(56,2.46,177.1,309.2)$ & $(40,2.40,176.9,306.2)$ & $(12,2.20,177.0,293.1)$ & $(8,2.13,177.0,259.5)$ & $(47,2.39,177.0,283.0)$ \\
\hline 0.15 & $(79,2.73,579.8,1258.1)$ & $(43,2.67,580.2,909.1)$ & $(15,2.50,580.8,885.7)$ & $(55,2.70,580.1,774.1)$ & $(11,2.49,580.3,869.6)$ \\
\hline 0.20 & $(68,2.68,375.6,700.2)$ & $(75,2.67,375.5,677.1)$ & $\begin{array}{c}(8,2.31,375.7,533.5) \\
n=75\end{array}$ & $(21,2.49,375.4,542.8)$ & $(2,2.10,375.6,425.3)$ \\
\hline 0.01 & $(70,1.91,102.1,7208.5)$ & $(81,2.22,102.3,997.6)$ & $(8,1.89,102.0,322.1)$ & $(86,2.46,102.2,247.6)$ & $(70,2.48,102.2,264.3)$ \\
\hline 0.02 & $(9,1.90,497.3,7429.5)$ & $(55,2.55,497.1,5228.6)$ & $(25,2.59,496.9,1532.7)$ & $(10,2.50,497.4,1443.0)$ & $(7,2.41,497.1,590.5)$ \\
\hline 0.05 & $(82,2.50,958.9,8512.0)$ & $(70,2.69,961.3,8794.1)$ & $(83,2.91,960.3,2925.2)$ & $(86,2.99,959.8,2226.7)$ & $(47,2.83,960.4,1407.9)$ \\
\hline 0.10 & $(68,2.65,405.0,832.2)$ & $(8,2.30,405.0,694.7)$ & $(96,2.70,405.4,776.9)$ & $(24,2.52,404.8,601.5)$ & $(8,2.35,404.9,538.8)$ \\
\hline 0.15 & $(29,2.53,321.9,545.6)$ & $(37,2.55,321.6,533.7)$ & $(6,2.25,321.8,420.2)$ & $(5,2.20,321.7,403.5)$ & $(97,2.72,321.8,428.5)$ \\
\hline 0.20 & $(70,2.85,818.1,1441.8)$ & $(37,2.74,817.2,1283.7)$ & $\begin{array}{c}(45,2.75,817.5,1240.3) \\
n=100\end{array}$ & $(56,2.79,817.5,1194.9)$ & $(73,2.85,817.8,1146.2)$ \\
\hline 0.01 & $(67,2.22,447.0,96358.4)$ & $(35,2.47,447.3,6529.2)$ & $(3,1.97,447.1,945.3)$ & $(7,2.37,447.2,849.6)$ & $(7,2.44,447.3,530.5)$ \\
\hline 0.02 & $(20,2.09,624.4,12820.0)$ & $(63,2.62,624.1,6276.6)$ & $(2,1.97,623.1,1380.3)$ & $(79,2.94,624.8,1138.3)$ & $(94,2.97,624.2,895.1)$ \\
\hline 0.05 & $(86,2.64,1124.5,6535.7)$ & $(80,2.73,1122.9,6048.6)$ & $(48,2.80,1123.7,3037.5)$ & $(74,2.96,1123.3,2434.8)$ & $(52,2.91,1123.2,1564.9)$ \\
\hline 0.10 & $(81,2.56,192.4,361.0)$ & $(9,2.22,192.3,278.4)$ & $(73,2.54,192.3,311.9)$ & $(35,2.42,192.3,266.6)$ & $(88,2.61,192.2,294.5)$ \\
\hline 0.15 & $(54,2.73,546.1,935.6)$ & $(61,2.74,545.3,870.6)$ & $(75,2.77,545.3,826.5)$ & $(32,2.63,545.5,713.7)$ & $(7,2.37,545.5,640.3)$ \\
\hline 0.20 & $(60,2.76,523.4,841.7)$ & $(41,2.69,523.2,747.5)$ & $(90,2.79,523.5,755.4)$ & $(57,2.73,523.3,692.1)$ & $(52,2.72,523.2,688.5)$ \\
\hline
\end{tabular}


In Table XI, we have computed the minimum values $m^{*}$ of $m$ for $n=\{25,50,75,100\}, p_{0}=\{0.01,0.02,0.05,0.1,0.15,0.2\}$, and chart parameters $\left(H_{n p}=2, K_{n p}=2.085\right),\left(H_{n p}=7, K_{n p}=2.322\right)$, and $\left(H_{n p}=47, K_{n p}=2.639\right)$, satisfying $\Delta=\frac{\left|A R L_{0, m^{*}}-A R L_{0, \infty}\right|}{A R L_{0, \infty}}<0.05$. As it can be noticed in Table $\mathrm{XI}$, depending on the values of $n$ and $p_{0}$, the value of $m^{*}$ satisfying $\Delta<0.05$ can be very large and, in some cases, larger than 10,000 .

In order to relax this constraint on the number of phase I samples, we suggest to compute alternative chart parameters $\left(H_{n p}^{\prime}\right.$, $K_{n p}$ ) for the synthetic $n p$ chart with estimated parameters, which take the value of $m$ into account and allow the in-control ARL value corresponding to the estimated parameters case to be as close as possible to the in-control ARL value corresponding to the known parameter case. These values are in Tables XII-XIV for $n=\{25,50,75,100\}, p_{0}=\{0.01,0.02,0.05,0.1,0.15,0.2\}, \quad$ and $m=\{10,20,50,100,200\}$. For example, in Table XII, if $n=75, p_{0}=0.05$, and $m=10$, the chart parameters are $\left(H_{n p}^{\prime}=26, K_{n p}^{\prime}=2.31\right)$, and, in this case, the in-control $A R L$ is $A R L_{0}=449.6\left(S D R L_{0}=1234.0\right)$. Referring to Table VIII, the in-control ARL corresponding to $m=\infty$ (i.e., the known parameter case) is $A R L_{0}=449.7$. Consequently, the use of the new chart parameters $\left(H_{n p}^{\prime}=26, K_{n p}^{\prime}=2.31\right)$ instead of $\left(H_{n p}=2, K_{n p}=2.085\right)$ allows one to obtain the same (or approximately the same) in-control ARL as for the known parameter case.

\section{Conclusions}

In this paper, we have compared the run length properties of the synthetic $c$ and $n p$ charts in both the known and estimated parameter cases. From this comparison, many interesting conclusions can be drawn. The first one is that for a particular value of $c_{0}$ or $\left(n, p_{0}\right)$, the in-control ARL values can be very different in the known and in the estimated parameter cases. Moreover, when $m$ increases, the difference in terms of in-control ARLs, between the known and the estimated parameter cases, tends to decrease but not always in a monotonic way, unlike the $\bar{X}$ chart, where this difference reduces monotonically with $m$. The second one is that depending on the values of $c_{0}$ or $\left(n, p_{0}\right)$, the in-control ARLs when $m<\infty$ are either smaller or larger than the in-control ARLs corresponding to $m=\infty$. This result is also quite different from what can be observed in the case of the $\bar{X}$ chart where the in-control ARL values corresponding to $m<\infty$ are always larger than the in-control ARL values corresponding to $m=\infty$. The third one is that if we want to use the chart parameters $(H=2, K=2.085),(H=7, K=2.322)$, and $(H=47, K=2.639)$ for the synthetic $c$ and $n p$ charts with estimated parameters, respectively, having run length performances close to that of the synthetic $c$ and $n p$ charts with known parameters, then we must pay the price to obtain a large number $m$ of phase I samples, sometimes larger than 10,000 . The fourth one is that the use of alternative chart parameters $\left(H^{\prime}, K^{\prime}\right)$ especially dedicated to the number $m$ of phase I samples allows the in-control ARL value corresponding to the estimated parameter case to be as close as possible to the in-control ARL value corresponding to the known parameter case.

\section{Acknowledgements}

This work is partially supported by the China Scholarship Council No [2011]6032. Support was also provided by the SARChI chair at the University of Pretoria, South Africa.

\section{References}

1. Montgomery DC. Introduction to Statistical Quality Control, 5th edn. Wiley: New York, 2004.

2. Wu Z, Spedding TA. A synthetic control chart for detecting small shifts in the process mean. Journal of Quality Technology 2000; 32(1):32-38.

3. Wu Z, Spedding TA. Implementing synthetic control charts. Journal of Quality Technology 2000; 32(1):75-78.

4. Calzada ME, Scariano SM. The robustness of the synthetic control chart to Non-normality. Communications in Statistics - Simulation and Computation 2001; 30(2):311-326.

5. Davis RB, Woodall WH. Evaluating and improving the synthetic control chart. Journal of Quality Technology 2002; 34(2):200-208.

6. Scariano SM, Calzada ME. A note on the lower-sided synthetic chart for exponentials. Quality Engineering 2003; 15(4):677-680.

7. Sim CH. Combined $\bar{X}$ and CRL charts for the gamma process. Computational Statistics 2003; 18(4):547-563.

8. Chen $\mathrm{FL}$, Huang HJ. A synthetic control chart for monitoring process dispersion with sample range. International Journal of Advanced Manufacturing Technology 2005; 26(7-8):842-851.

9. Huang HJ, Chen FL. A synthetic control chart for monitoring process dispersion with sample standard deviation. Computers \& Industrial Engineering 2005; 49(2):221-240.

10. Chen FL, Huang HJ. Variable sampling interval synthetic control charts for jointly monitoring process mean and standard deviation. International Journal of Industrial Engineering: Theory Applications and Practice 2006; 13(2):136-146.

11. Costa AFB, Rahim MA. A synthetic control chart for monitoring the process mean and variance. Journal of Quality in Maintenance Engineering 2006; 12(1):81-88.

12. Bourke PD. Performance comparisons for the synthetic control chart for detecting increases in fraction nonconforming. Journal of Quality Technology 2008; 40(4):461-475.

13. Khoo MBC, Wu Z, Atta AMA. A synthetic control chart for monitoring the process mean of skewed populations based on the weighted variance method. International Journal of Reliability, Quality and Safety Engineering 2008; 15(3):217-245.

14. Castagliola P, Khoo MBC. A synthetic scaled weighted variance control chart for monitoring the process mean of skewed populations. Communications in Statistics - Simulation and Computation 2009; 38(8):1659-1674.

15. Aparisi F, de Luna MA. Synthetic-control charts optimized for in-control and out-of-control regions. Computers \& Operations Research 2009; 36(12):3204-3214. 
16. Scariano SM, Calzada ME. The generalized synthetic chart. Sequential Analysis 2009; 28(1):54-68.

17. Calzada ME, Scariano SM. A Synthetic Control Chart for the Coefficient of Variation. Journal of Statistical Computation and Simulation 2013; 83(5):851-865.

18. Zhang Y, Castagliola P, Wu Z, Khoo MBC. The synthetic $\bar{X}$ chart with estimated parameters. IIE Transactions 2011; 43(9):676-687.

19. Chakraborti S, Human SW, Graham MA. Phase I statistical process control charts: an overview and some results. Quality Engineering 2009; 21(1):52-62.

20. Jensen WA, Jones-Farmer LA, Champ CW, Woodall WH. Effects of parameter estimation on control chart properties: a literature review. Journal of Quality Technology 2006; 38(4):349-364.

21. Quesenberry CP. The effect of sample size on estimated limits for $\bar{X}$ and X control charts. Journal of Quality Technology $1993 ; \mathbf{2 5}(4): 237-247$.

22. Del Castillo E. Evaluation of the run length distribution of $\bar{X}$ charts with unknown variance. Journal of Quality Technology $1996 ; \mathbf{2 8}(1): 116-122$.

23. Chen G. The mean and standard deviation of the run length distribution of $\bar{X}$ charts when control limits are estimated. Statistica Sinica 1997; 7(3):789-798.

24. Ghosh BK, Reynolds Jr MR., Van Hui Y. Shewhart $\bar{X}$ charts with estimated variance. Communications in Statistics - Theory and Methods 1981 ; 10(18):1797-1822.

25. Jones LA, Champ CW, Rigdon SE. The performance of exponentially weighted moving average charts with estimated parameters. Technometrics $2001 ; \mathbf{4 3}(2): 156-167$.

26. Nedumaran G, Pignatiello, Jr JJ. On estimating $\bar{X}$ control chart limits. Journal of Quality Technology 2001; 33(2):206-212.

27. Jones LA. The statistical design of EWMA control charts with estimated parameters. Journal of Quality Technology 2002; 34(3):277-288.

28. Yang Z, Xie M, Kuralmani V, Tsui K. On the performance of geometric charts with estimated control limits. Journal of Quality Technology 2002; 34(4):448-458.

29. Champ CW, Jones LA. Designing phase I $\bar{X}$ charts with small sample sizes. Quality and Reliability Engineering International 2004; 20(5):497-510.

30. Chakraborti S. Parameter estimation and design considerations in prospective applications of the $\bar{X}$ chart. Journal of Applied Statistics 2006 ; 33(4):439-459.

31. Jensen WA, Bryce GR, Reynolds Jr MR. Design issues for adaptive control charts. Quality and Reliability Engineering International 2008; 24(4):429-445.

32. Zhang Y, Castagliola P. Run rules $\bar{X}$ charts when process parameters are unknown. International Journal of Reliability, Quality and Safety Engineering 2010; 17(4):381-399.

33. Hillier FS. $\bar{X}$ - And R - chart control limits based on a small number of subgroups. Journal of Quality Technology $1969 ; \mathbf{1}(1): 17-26$.

34. Hawkins DM. Self-starting cusum charts for location and scale. The Statistician 1987; 36(4):299-316.

35. Quesenberry CP. SPC Q charts for start-up processes and short or long runs. Journal of Quality Technology 1991; 23(3):213-224.

36. Chen G. The run length distributions of the $R, S$ and $S^{2}$ control charts when $\sigma$ is estimated. The Canadian Journal of Statistics 1998; 26(2):311-322.

37. Maravelakis PE, Panaretos J, Psarakis S. Effect of estimation of the process parameters on the control limits of the univariate control charts for process dispersion. Communications in Statistics - Simulation and Computation 2002; 31(3):443-461.

38. Maravelakis P, Castagliola P. An EWMA chart for monitoring the process standard deviation when parameters are estimated. Computational Statistics \& Data Analysis 2009; 53(7):2653-2664.

39. Castagliola P, Celano G, Chen G. The exact run length distribution and design of the $S^{2}$ chart when the in-control variance is estimated. International Journal of Reliability, Quality and Safety Engineering 2009; 16(1):23-38.

40. Castagliola P, Maravelakis P. A CUSUM control chart for monitoring the variance when parameters are estimated. Journal of Statistical Planning and Inference 2011; 141(4):1463-1478.

41. Braun WJ. Run length distributions for estimated attributes charts. Metrika 1999; 50(2):121-129.

42. Castagliola P, Wu S. Design of the $c$ and $n p$ charts when the parameters are estimated. International Journal of Reliability, Quality and Safety Engineering 2012; 19(2):1250010.

43. Chakraborti S, Human SW. Parameter estimation and performance of the $p$-chart for attributes data. IEEE Transactions in Reliability 2006; 55(3):559-566.

44. Chakraborti S, Human SW. Properties and performance of the c-chart for attributes data. Journal of Applied Statistics 2008; 35(1):89-100.

45. Del Castillo E. Discussion. Journal of Quality Technology 1995; 27(4):316-321.

\section{Authors' biographies}

Philippe Castagliola graduated (PhD 1991) from the UTC (Université de Technologie de Compiègne, France). He is currently professor at the Université de Nantes, Institut Universitaire de Technologie de Nantes, France, and he is also a member of the IRCCyN (Institut de Recherche en Communications et Cybernétique de Nantes), UMR CNRS 6597. He is editor for the Journal of Quality Technology and Quantitative Management and associate editor for the International Journal of Reliability, Quality and Safety Engineering. His research activity includes developments of new SPC techniques (non normal control charts, optimized EWMA type control charts, control charts with estimated parameters, multivariate capability indices, monitoring of batch processes,...).

Shu WU has a MSc from the School of Logistics Engineering at Wuhan University of Technology, Wuhan, China. She is currently a PhD student at the Université de Nantes, France. Her main areas of interest are in new Statistical Quality Control techniques.

Michael B. C. Khoo is a Professor in the School of Mathematical Sciences, Universiti Sains Malaysia (USM). He received his Ph.D. in Applied Statistics in 2001 from USM. His research interest is in Statistical Process Control. He is a member of the American Society for Quality and serves as a member of the editorial boards of several international journals.

Subhabrata Chakraborti is Professor of Statistics and Robert C. and Rosa P. Morrow Faculty Excellence Fellow at the University of Alabama, U.S.A. He is a Fellow of the American Statistical Association, an elected member of the International Statistical Institute and has been a Fulbright Senior Scholar. Professor Chakraborti has authored and co-authored over one hundred publications in a variety of international journals and outlets. He is the co-author of the book Nonparametric Statistical Inference, fifth edition (2010), published by CRC Press/Taylor and Francis. His current research interests include applications of statistical methods, particularly nonparametric statistical methods, to the area of statistical process control. He is currently serving his fifteen year term as an Associate Editor of Communications in Statistics. He is a member of the American Statistical Association, the International Statistical Association and the Institute of Mathematical Statistics. 